\title{
1 Establishing Probiotic Saccharomyces boulardii as a Model 2 Organism for Synthesis and Delivery of Biomolecules
}

3

4 Deniz Durmusoglu ${ }^{1,+}$, Ibrahim Al'Abri ${ }^{1{ }^{+}}$, Scott P. Collins ${ }^{1}$, Chase Beisel ${ }^{1}$, Nathan 5 Crook $^{1^{*}}$

6

$7 \quad{ }^{1}$ Department of Chemical and Biomolecular Engineering, North Carolina State University, Raleigh, North

8 Carolina, USA.

$9 \quad$ These authors contributed equally to this work

10 *To whom correspondence should be addressed: nccrook@ncsu.edu 


\section{Abstract}

13 Saccharomyces boulardii is a widely used yeast probiotic which can counteract various

14 gastrointestinal disorders ${ }^{1}$. As a relative of Saccharomyces cerevisiae, S. boulardii exhibits rapid

15 growth and is easy to transform ${ }^{2}$ and thus represents a promising chassis for the engineered

16 secretion of biomolecules. To establish S. boulardii as a platform for delivery of biomolecules to

17 the mammalian gut, we measured the amount and variance in protein expression enabled by

18 promoters, terminators, selective markers, and copy number control elements in this organism.

19 These genetic elements were characterized in plasmidic and genomic contexts, revealing

20 strategies for tunable control of gene expression and CRISPR-mediated genome editing in this

21 strain. We then leveraged this set of genetic parts to combinatorially assemble pathways

22 enabling a wide range of drug and vitamin titers. Finally, we measured S. boulardii's residence

23 time in the gastrointestinal tracts of germ-free and antibiotic-treated mice, revealing the

24 relationships between dosing strategy and colonization level. This work establishes S. boulardii

25 as a genetically tractable commensal fungus and provides a set of strategies for engineering $S$.

26 boulardii to synthesize and deliver biomolecules during gut colonization. 


\section{Introduction}

29 Next-generation probiotics offer unique attributes for the delivery of biomolecules to the gut ${ }^{3}$.

30 By leveraging decades of work in metabolic engineering ${ }^{4}$, engineered probiotics have enabled

31 the conversion of unused dietary material to beneficial products for the host ${ }^{5-7}$. Furthermore,

32 the ongoing development of synthetic biological sensors advance the concept of drug synthesis

33 tailored to the severity and location of disease within the host ${ }^{8-11}$. Coupled with existing

34 manufacturing expertise in food science and infrastructure in place for distributing probiotics,

35 engineered probiotics promise to substantially reduce the costs associated with production of

36 drug molecules ${ }^{12}$. All engineered probiotics described to date have been members of the

37 bacterial domain of life due to their high numerical abundance in the gut ${ }^{13,14}$ and ease of

38 engineering. ${ }^{9,15}$ For example, Escherichia coli Nissle 1917 has been engineered to treat diseases

39 such as phenylketonuria $(\mathrm{PKU})^{15,16}$, hyperammonemia ${ }^{17}$, and cancer $^{18}$, or eliminate pathogens

40 such as Pseudomonas aeruginosa ${ }^{19}$. In addition to these advantages, the limitations to bacterial

41 probiotics include susceptibility to antibiotics, ${ }^{20}$ predation by bacteriophage known to be highly

42 abundant in the human gut, ${ }^{21}$ and difficulty in producing high levels of post-translationally-

43 modified proteins ${ }^{22}$.

45 In addition to bacteria, a diverse fungal population also exists in the human gastrointestinal tract

$46(\mathrm{GIT})^{23-26}$. While the numerical abundance of fungal cells in the GIT is much lower than bacterial

47 cells, fungal cells are on average 100-fold larger, indicating that their biomass and role in the gut

48 may be larger than metagenomic surveys indicate ${ }^{25,27}$. Recent studies have shown that while 
commensal fungi play important roles in the development of inflammatory bowel diseases, they also provide protective heterologous immunity to pathogenic organisms by training the immune

51 system ${ }^{28,29}$. Furthermore, fungi are not susceptible to bacteriophage predation and are easily

52 engineered to secrete high titers of proteins that are post-translationally modified. Thus, in this

53 study, we established a pipeline to engineer the only eukaryotic probiotic that is approved by the

54 FDA: Saccharomyces boulardii ${ }^{30}$.

55

S. boulardii is a non-pathogenic yeast that was originally isolated from lychee and mangosteen in

571923 and has since been used to treat ulcerative colitis, diarrhea, and recurrent Clostridium

58 difficile infection ${ }^{1,31}$. S. boulardii is closely related to the famous budding yeast Saccharomyces

59 cerevisiae, indicating that it may be similarly amenable to engineering for the production of

60 biomolecules, including biologics requiring post-translational modifications ${ }^{2,32}$. Supporting this,

61 S. cerevisiae expression vectors can be transformed and propagated in S. boulardii, and

62 CRISPR/Cas9-mediated genome editing is functional in both strains ${ }^{33}$. However, compared to $S$.

63 cerevisiae, S. boulardii better tolerates low $\mathrm{pH}$ environments and grows more rapidly at human

64 body temperature $32,34,35$. Given its regulatory status, genetically tractability, and well-studied

65 yeast relative, S. boulardii is uniquely poised as a potential model chassis for the delivery of

66 therapeutics to the human gut. Indeed, S. boulardii has been engineered to secrete human

67 synthetic lysozyme ${ }^{33}$ and HIV-1 Gag ${ }^{36}$ in cell culture, as well as IL-10 in an IBD mouse model ${ }^{37}$.

68 However, while the rules governing high-level biomolecule production in S. cerevisiae have been

69 extensively studied, these rules remain to be defined in culture- and gut-resident S. boulardii. In

70 this work, we sought to develop a quantitative framework for engineering S. boulardii for in situ 
71 production and delivery of therapeutics to the mammalian gut. We began by assaying the ability

72 of a library of S. cerevisiae genetic parts ${ }^{38}$ to drive gene expression from various plasmidic and

73 genomic contexts in S. boulardii. We then applied these genetic parts to rapidly construct and

74 identify S. boulardii strains exhibiting high levels of vitamin ( $\beta$-carotene) or drug (violacein)

75 production. Finally, we measured the residence time of $S$. boulardii in the mouse gut. Taken

76 together, this work demonstrates the promise of, and details a set of procedures for engineering,

77 S. boulardii for producing biomolecules in the gut.

79 Results

80 Selective marker and plasmid origin tune gene expression over a wide range in $S$.

81 boulardii

82 Plasmids enable rapid prototyping of synthetic constructs in many organisms, including yeast.

83 The tunability of gene expression on plasmids is due in part to plasmid-specific copy number

84 control sequences, which in yeast have been shown to include the origin of replication (origin)

85 and selectable marker (marker) ${ }^{39}$. We therefore first sought to understand the interplay between

86 origin and marker in determining the expression of a model fluorescent reporter gene. An

87 optimal reporter gene, when expressed, should yield detection signals well above the

88 background signal produced by non-expressing strains in order to precisely resolve gene

89 expression levels ${ }^{40}$. Therefore, we first measured the cell fluorescence conferred by three

90 proteins (Venus, mTurquoise2, mRuby2) when expressed by the TDH3 promoter and the TDH1 
91 terminator on a plasmid harboring a URA3 marker and the $2 \mu$ origin. Fluorescence levels were

92 measured at log phase using flow cytometry. In all experiments, we normalized the resulting

93 fluorescence levels to those of a strain harboring an empty vector. Venus displayed the highest

94 normalized fluorescence (1080-fold) (Supplementary Figure S1). However, Venus fluorescence

95 also exhibited high cell-to-cell variability compared to that for $m$ Ruby 2 and $m$ Turquoise2, and its

96 fluorescence distribution was non-gaussian, suggesting that Venus would not be suitable for

97 measuring fine differences in expression. On the other hand, $m R u b y 2$ showed the second highest

98 normalized fluorescence (450-fold) and exhibited a more gaussian fluorescence distribution.

99 Because mRuby2 exhibited a 2.6-fold higher normalized fluorescence than mTurquoise2, we

100 decided to proceed with mRuby2 for further analysis of synthetic construct performance in $S$.

101 boulardii.

102

103 We generated expression cassettes containing the TDH3 promoter ( $p T D H 3), m R u b y 2$, and the

104 TDH1 terminator (tTDH1) in combination with 3 auxotrophic markers (URA3, HIS3 and TRP1) or

105 four antifungal markers (geneticin resistance (KanMX), zeocin resistance (ZeoR), nourseothricin

106 resistance (NatR), and hygromycin resistance (HygR)) and two origins-of-replication (CEN6/ARS4

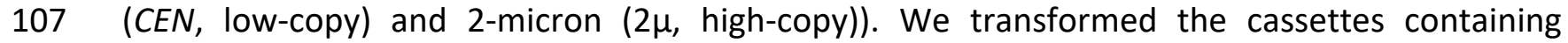

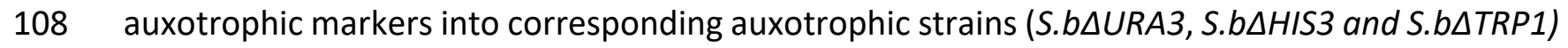

109 and antifungal markers into wild-type strain. We achieved a maximum normalized fluorescence

110 of 400 using URA3 and $2 \mu$ (Figure 1a, Supplementary Figure S2a). Among antifungal markers,

111 HygR coupled with the $2 \mu$ origin yielded the highest fluorescence (Figure 1a). Other 112 marker/origin combinations yielded a 100-fold variation in fluorescence (Figure 1a, 
113 Supplementary Figure S2). This large range in expression levels for constructs containing the

114 same promoter and terminator indicated that, as in S. cerevisiae, plasmid origin and marker have

115 significant effects on gene expression in S. boulardii.

117 The impact of origin on gene expression is marker-dependent in S. boulardii.

118 We next asked whether certain markers or origins had consistent effects on fluorescence. For

119 auxotrophic markers, $2 \mu$ plasmids consistently yielded higher fluorescence than CEN plasmids

120 (Two-way ANOVA, $\mathrm{p}<0.05$ ) (Figure 1a). This is consistent with prior work in S. cerevisiae, which

121 demonstrated that $2 \mu$ plasmids with auxotrophic markers are maintained at 28-58 copies per

122 cell, whereas CEN plasmids are maintained at only 4-8 copies per cell ${ }^{39}$. However, with the

123 exception of HygR (Two-way ANOVA, $\mathrm{p}<0.0001$ ), the fluorescence conferred by plasmids

124 containing antifungal markers was not impacted by the choice of origin (Two-way ANOVA,

$125 \mathrm{p}>0.05)$. For the CEN origin, using NatR at the maximum antifungal concentration yielded the

126 highest fluorescence level. This level was significantly higher expression than those conferred by

127 the HIS3, ZeoR, KanMX, or HygR markers (Two-way ANOVA, p<0.05). However, for the $2 \mu$ origin,

128 using any of the auxotrophic markers gave gene expression levels higher than any antifungal

129 marker (Two-way ANOVA, $\mathrm{p}<0.0001$ ). Taken together, our data indicate that gene expression

130 conferred by plasmids containing auxotrophic markers (but not antifungal markers) is sensitive

131 to the choice of origin, and that selective marker significantly impacts gene expression for both

$1322 \mu$ and CEN origins. 


\section{Plasmidic gene expression noise is marker and origin dependent in S. boulardii}

135 Cell-to-cell variability in gene expression can have dramatic impacts on overall production

136 titers ${ }^{41}$. Therefore, it is desirable to design engineered probiotics to exhibit minimal gene

137 expression noise. Previous work characterizing gene expression noise in S. cerevisiae showed that

138 cell variability varies as a function of promoter, terminator, origin, and selective marker ${ }^{42}$. We

139 thus calculated the population-level coefficient of variance (CV) in the fluorescence values we

140 obtained for S. boulardii. We found that marker, origin, and marker-origin interactions had

141 significant impacts on expression noise (Two-way ANOVA, $\mathrm{p}<0.05$ ). Specifically, CEN plasmids

142 exhibited less cell-cell variability than $2 \mu$ plasmids (Two-way ANOVA, $p<0.05$ ). Additionally,

143 antifungal markers exhibited less noisy expression than auxotrophic markers (Two-way ANOVA,

$144 \mathrm{p}<0.05)$. These data suggest differences in the ways that the auxotrophic and antifungal markers

145 interact with origins of replication to control gene expression, and point to design strategies

146 which reduce plasmid-based gene expression variance.

148 S. cerevisiae promoters largely maintain their relative activities in S. boulardii.

149 Promoter sequences are frequently employed to tune the expression of individual genes in

150 yeast ${ }^{43,44}$. Here, we quantified the transcriptional strength of 18 constitutive $S$. cerevisiae

151 promoters driving the expression of a fluorescent protein in S. boulardii. Each of these promoters

152 had a homolog in S. boulardii, with percent nucleotide identities ranging from $100 \%$ to $97 \%$

153 (Supplementary Table S1). In these experiments, vectors contained the $2 \mu$ origin and the URA3

154 auxotrophic marker, which maximized the range over which changes to fluorescence could be

155 detected (Figure 1a). Each promoter drove the expression of mRuby2 and transcription was 
terminated by $t T H D 1$. These constructs were transformed into $S$. boulardii and fluorescence

157 levels were measured at log phase via flow cytometry. Each promoter yielded different mean

158 fluorescence values (Two-way ANOVA, $p<0.0001$ ), and these promoters collectively modulated

159 fluorescence levels over a 40-fold range (Figures 1b, S3). While the relative activities of 17 of the

16018 promoters in S. boulardii were similar to reported values in S. cerevisiae, one promoter

161 (pALD6) showed 10-fold higher activity in S. boulardii than in S. cerevisiae (Supplementary Figure

162 S4). While the precise role of $A L D 6$ in the probiotic properties of S. boulardii is unknown, Ald6p

163 is involved in the detoxification of aldehyde molecules to their corresponding acid in $S$.

164 cerevisiae $^{45}$. Collectively, this data curates a set of promoters enabling precise tuning of gene

165 expression in S. boulardii, and indicates that constructs developed for S. cerevisiae will exhibit

166 similar gene expression profiles in S. boulardii.

Terminators modulate gene expression in a promoter-dependent manner in $S$.

169 boulardii

170 Terminators regulate expression in yeast by modulating mRNA stability, translational efficiency

171 and mRNA localization ${ }^{46}$. Furthermore, a terminator's effect on expression can depend on the

172 strength of the promoter or the sequence of the expressed gene ${ }^{47,48}$. Therefore, we were

173 interested in defining the effect of terminator choice on gene expression in S. boulardii. We

174 cloned 6 terminators (tENO1, tENO2, tSSA1, tPGK1, tADH1, and $t T D H 1$ ) into mRuby2 expression

175 constructs driven by strong ( $p T D H 1)$, medium ( $p R N R 1)$, or weak ( $p R E V 1)$ promoters. We found

176 that these terminators could modulate gene expression by $1.77,1.81$, and 1.64 -fold in constructs

177 containing $p T D H 1, p R N R 1$, or $p R E V 1$, respectively, effectively increasing the expression range 
178 beyond that enabled by varying the promoter alone (40-fold, Figure $1 \mathrm{~b}$ ). In particular, using a

179 strong promoter (TDH3) with the $T D H 1$ terminator yielded 80-fold higher expression than a weak

180 promoter (REV1) with the SSA1 terminator. Relative to tSSA1 and tTDH1, we found that tENO1,

181 tENO2, $t A D H 1$ and $t P G K 1$ significantly decreased gene expression in constructs containing the

182 strong $p T D H 3$ promoter (Two-way ANOVA, $\mathrm{p}<0.005$ ) while for $p R N R 1$ and $p R E V 1$, the differences

183 in gene expression we observed among terminators were not statistically significant (Two-way

184 ANOVA, $p>0.05$ ) (Figure 1c, S5). Taken together, this data suggests that varying the terminator is

185 a valuable way to fine-tune gene expression, particularly when certain regulatory characteristics

186 of the promoter (e.g. responsiveness to growth phase or gut conditions) must be maintained.

188 The $S$. boulardii genome can be efficiently modified by CRISPR-Cas genome editing

190 Genomic integration of synthetic constructs can sidestep issues associated with the use of

191 plasmids in the gut. Such issues include plasmid instability if no selective pressure is present,

192 potential spread to other microbes, collateral damage to the microbiota if antimicrobials are used

193 to select for plasmid maintenance, and the increased metabolic burden associated with the

194 maintenance of multicopy plasmids. However, different genomic regions are known to support

195 varying levels of synthetic construct expression, potentially due to nucleosome positioning ${ }^{49}$, and

196 integrating material into the genome can be especially difficult for diploid and polyploid

197 organisms ${ }^{50}$. 
To characterize and overcome inefficiencies associated with genome integration, we compared integration) and genome editing assisted with the CRISPR nucleases SpCas9 or LbCas12a. We

202 tested the ability of these methods to integrate a linear expression cassette containing 203 mTurquoise2 in three different loci (INT1, 4 and 5) via colony PCR. In the case of dsDNA 204 integration, 4/12 transformants did not contain the expected mTurquoise 2 insertion, while 7/12 205 colonies yielded multiple PCR fragments (Supplementary Figure S6). Sequencing revealed that 206 the larger fragment corresponded to the desired integration, while the shorter fragment 207 corresponded to the native genomic locus. This result is likely due to the fact that the S. boulardii 208 genome is diploid, and that integration of one copy of the construct is sufficient to confer uracil 209 prototrophy.. Therefore, we tested whether CRISPR-based editing tools could achieve more 210 complete genome editing in S. boulardii. We reasoned that since CRISPR-Cas systems enhance

211 the proportion of double-stranded breaks in all copies of the targeted sequence, editing of all

212 chromosomal copies would be a preferred survival strategy. Three sgRNAs for SpCas9 and three 213 gRNAs for LbCas12a were designed for each integration site. Targeted loci were followed by a 5'214 NGG-3' protospacer adjacent motif (PAM) for SpCas9 and preceded by a 5'-TTTV-3' PAM (V = A, 215 C, G) for LbCas12a. Supplementary Figure S7c-f shows the plasmid designs we used for CRISPR216 based editing, Supplementary Table S2 shows integration locations (INT1-INT5) in S. boulardii's 217 genome, and Supplementary Table S3 shows the guide RNA sequences we used.

219 We tested the editing efficiency at each integration site (using a single guide RNA per experiment) 
221 fluorescent colonies we observed for each editing method. Colony PCR of 4 random fluorescent

222 transformants from each experiment revealed a single band corresponding to either the edited

223 or the unedited version of the locus (Supplementary Figure S8). Altogether, we were able to

224 generate genome integrants with $82-88 \%$ efficiency for SpCas9 and 93-99\% efficiency for

225 LbCas12a in S. boulardii (Figure 2a, Supplementary Table S4). Thus, these results indicate that

226 CRISPR/LbCas12a-assisted genome editing has $>10 \%$ and $>50 \%$ higher editing efficiency than

227 CRISPR/SpCas9 and dsDNA integration, respectively, and is therefore a promising choice for

228 future editing in this strain.

Integration locus has a minor effect on gene expression, relative to promoter

231 choice

232 To determine the extent of locus-dependent effects on gene expression in S. boulardii, we

233 integrated constructs driving mRuby2 or mTurquoise 2 expression into five different locations in

234 the S. boulardii genome (Supplementary Table S2) using LbCas12a. These genes were regulated

235 by $p T D H 3$ and $t T D H 1$, and constructs contained URA3 to select for edited strains (Supplementary

236 Figure S7b). The mRuby2 and mTurquoise2 fluorescent proteins are sequence-divergent, with

237 only $30 \%$ amino acid identity. These two proteins therefore enabled a cursory view into the

238 generalizability of these patterns across genes. Across integration sites, the expression of

239 mRuby2 and mTurquoise2 varied 3.4-fold and 2.6-fold, respectively (Figure 2b, S9). INT2

240 supported the lowest expression for both genes, while INT4 supported the highest expression

241 levels for mRuby2 and INT5 supported the highest expression level for mTurquoise2. We next

242 asked whether differences in promoter strength observed in the plasmid-based system would be 
243 similar for genomically-integrated constructs. We therefore expressed $m$ Ruby 2 and $m$ Turquoise2

244 from two additional promoters in INT4: $p R N R 1$ and $p R E V 1$. Intriguingly, genomic mRuby2

245 expression varied by 5.9-fold between $p T D H 3$ and $p R N R 1$, compared with 2.8-fold for the same

246 construct on a $2 \mu$ plasmid. Conversely, genomic mRuby2 expression varied by 15.3-fold between

247 PTDH3 and $p R E V 1$, compared to 31.2-fold for the same promoters used on a $2 \mu$ plasmid (Figure

248 2c). This could indicate that gene expression from high-copy plasmids saturates at high promoter

249 strengths, or that promoter strength varies with copy number due to transcription factor dilution.

250 Together, this work establishes a pipeline for efficient genome integration in S. boulardii and

251 identifies regions of the $S$. boulardii genome enabling high-level expression of synthetic

252 constructs. Further, these data show that the impact of integration locus on gene expression is

253 relatively small in S. boulardii compared to that of the promoter.

254

255 S. boulardii facilitates high-throughput metabolic engineering through in vivo

256 combinatorial assembly of multi-enzyme pathways

257 Having found that S. boulardii could support genome integration via homologous recombination,

258 we asked if recombination efficiencies were sufficient for in vivo assembly of biosynthetic

259 pathways. In S. cerevisiae, combinatorial in vivo assembly is a facile technique for rapid

260 optimization of metabolic pathways ${ }^{51-53}$. Large scale assembly of common heterologous

261 metabolic pathways directly in S. boulardii would simplify strain development and might reveal

262 the potential for S. boulardii as a host for biomolecule production. 
264 We implemented golden gate assembly to streamline in vivo assembly workflows in S. boulardii.

265 Namely, we started from our characterized promoter, coding sequence (CDS), and terminator

266 parts and assembled pathways directly in yeast with minimal subcloning (Figure 3a). Our

267 assembly strategy involved two steps: a golden gate assembly step to assemble a 9-member

268 gene-level library of promoter-CDS-terminator transcriptional units for each gene in the pathway

269 of interest and in vivo homologous recombination to assemble the gene-level libraries created in

270 step 1 into pathways, as shown in Figure 3a. Each gene-level library member differed only in the

271 promoter driving gene expression. These transcriptional units are flanked with homology arms

272 which facilitate in vivo assembly. These gene-level libraries are then PCR-amplified directly from

273 the Golden Gate reaction mixture. Purified amplicons are transformed into S. boulardii, where

274 flanking sequences direct homology-dependent assembly of the final pathway with one variant

275 of each transcriptional unit. We used this method to construct $\beta$-carotene and violacein synthesis

276 pathways, which provided a direct colorimetric readout of assembly efficacy (Supplementary

277 Figures S10a and S10b). Supplementary Figures S10c and S10d show spotted cultures of 28 library

278 members from the $\beta$-carotene pathway and 24 library members from the violacein pathway.

279 Colony sequencing revealed that each strain harbored a plasmid containing the expected

280 pathway topology, with each gene driven by one of the 10 selected promoters and the desired

281 terminator.

282

283 We next focused on those strains exhibiting qualitatively stable pigment levels over three rounds

284 of growth of solid media. 13 out of 28 strains and 8 out of 24 strains stably produced $\beta$-carotene

285 and violacein molecules, respectively. We determined the identity of the promoters driving each 
gene in these strains. We also quantified the amount of $\beta$-carotene produced by these strains to

287 understand how different combinations of promoters regulate biomolecule production. Figures

$2883 \mathrm{~b}$ and S11 show the strength of the promoters comprising each assembled pathway and amount

289 of $\beta$-carotene or violacein produced by each assembled pathway. Interestingly, we found that

290 strains with the highest qualitative color intensity and quantitative $\beta$-carotene concentration did

291 not harbor strong promoters for all the genes in the pathway. In fact, many high producers

292 contained moderate or low-strength promoters in at least one of their genes. Additionally, we

293 observed cases where multiple pathway genes in a high-producing strain were driven by the

294 same promoter. This suggests that product titers in these strains were not sufficiently high to

295 select for the emergence of non-producers via homologous recombination.

297 The optimal expression levels for several genes in the $\beta$-carotene are not obvious from the 298 biosynthetic reaction map ${ }^{54}$. HMG1 diverts flux from cellular acetyl-coA pools into the $\beta$-carotene 299 pathway, thereby reducing the amount of acetyl-coA available for other processes. crtYB can convert lycopene into the desired product $\beta$-carotene, but can also convert neurosporene (a 301 precursor to lycopene) into the side product 7,8-dihydro- $\beta$-carotene ( $\beta$-zeacarotene). crtl 302 operates on several pathway intermediates, including phytoene, $\zeta$-carotene, and 303 neurosporene ${ }^{55}$. Figure $3 b$ shows substantial variations in $\beta$-carotene titer depending on the 304 expression level of these genes. As complex tradeoffs between gene expression and pathway 305 output will likely also be present in engineered strains colonizing the gut, combinatorial assembly 306 is useful for rapidly generating strain designs for subsequent in vivo testing or screening. Our 307 results reveal that high biomolecule titers can be achieved via expression tuning, confirming 
previous results from other methods for pathway optimization in S. cerevisiae $52,53,56$. Moreover, as in S. cerevisiae, large combinatorial pathway libraries can be easily generated in S. boulardii, enabling rapid optimization of biomolecule titers.

\section{The Mouse Microbiota Restricts S. boulardii Colonization}

Host immunity, intestinal peristalsis, inter-microbial competition, and nutrient availability shape

314 the residence time of microbes in the gut. To parse this complexity, gnotobiotic mouse models

315 are attractive to explore the behavior of microbes in defined gut ecologies. Thus, we first measured S. boulardii's residence time in the germ-free gut by delivering S. boulardii $\left(10^{8}, 10^{7}\right.$, or

$31710^{6}$ colony-forming units) to germ-free mice via oral gavage (Supplementary Figure S12). No

318 deleterious effects on mouse health were apparent during the experiment. While all dosages

319 yielded similar fecal loads of S. boulardii through day 5 ( $3 \times 10^{6} \mathrm{CFU} / \mathrm{g}$ feces), we found that when

320 mice were provided at least $10^{8}$ CFUs, consistent fecal loads of $10^{7} \mathrm{CFU} / \mathrm{g}$ S. boulardii were

321 maintained for over 30 days. On the other hand, mice gavaged with $10^{6}$ and $10^{7}$ CFUs showed 3-

322 fold lower fecal titers starting on day 7. Thus, the initial dose of S. boulardii impacts its fecal titer

323 and residence time in germ-free mice. Furthermore, in the absence of microbial competitors, $S$.

324 boulardii is capable of long-term (>30 days) colonization of the GIT.

326 We next asked whether the presence of microbial competitors would modulate the gut residence

327 time of S. boulardii. To answer this, we turned to conventionally-raised mice treated with 328 antibiotics ${ }^{57}$. In these experiments, we were initially unable to track S. boulardii titers via plating 329 because other native gut fungi outgrew S. boulardii on YPD plates containing $0.125 \mathrm{mg} / \mathrm{ml}$ 
330 penicillin and $0.25 \mathrm{mg} / \mathrm{ml}$ streptomycin. Therefore, we hypothesized that integration of an

331 antifungal resistance gene into the $S$. boulardii genome would allow easier identification via

332 plating on media containing antifungals. Based on the observed minimum inhibitory

333 concentrations (MICs) of various antifungals against S. boulardii (Supplementary Figure S13), we

334 integrated the nourseothricin resistance gene (NatR) into the S. boulardii genome. We found that

335 NatR-containing S. boulardii was the only microbe from mouse feces able to grow on YPD plates

336 containing $50 \mu \mathrm{g} / \mathrm{ml}$ nourseothricin, $0.125 \mathrm{mg} / \mathrm{ml}$ Penicillin and $0.25 \mathrm{mg} / \mathrm{ml}$ streptomycin (data

337 not shown). We then provided conventional mice with $1 \mathrm{mg} / \mathrm{ml}$ penicillin and $2 \mathrm{mg} / \mathrm{ml}$

338 streptomycin in their drinking water for four days, after which antibiotics treatment ceased and

$33910^{8} \mathrm{CFU}$ of S. boulardii was delivered once per day for three days. Under this regimen (Treatment

340 1, Figure 4b), we found that $S$. boulardii titers fell to undetectable levels within 48 hours of the

341 last gavage (Figure 4D). On the other hand, when antibiotic treatment was maintained over the

342 course of the experiment (Treatment 2, Figure 4c), the titer and residence time of S. boulardii

343 both increased, enabling $S$. boulardii to remain present in the gut one additional day after the

344 last gavage (Figure 4D). These results are in line with previous S. boulardii colonization studies

345 performed in mice colonized by a human microbiota ${ }^{58}$. Taken together, these experiments

346 indicate that competitive microbial growth reduces the residence time of $S$. boulardii in the

347 mouse gut. In these contexts, S. boulardii-mediated drug delivery will only occur during and for

348 several days after probiotic administration, thereby minimizing dosage outside of desired

349 therapeutic timescales. 


\section{Conclusion}

352 The gut and the microbes that live within it play a major role in host health, and as such are

353 promising targets for manipulation via small molecule or biologic drugs. Unfortunately, delivering

354 drugs to this environment remains challenging due to the digestive action of the host, especially

355 for protein-based therapies. Due to its probiotic properties and similarity to the

356 biomanufacturing workhorse organism S. cerevisiae, we characterized the ability of S. boulardii

357 to be engineered to secrete biomolecules in the mammalian gut. We first characterized the effect

358 of different plasmid-based and genomic sequences on the expression of synthetic constructs. We

359 next established efficient genome editing tools for this strain and revealed the effects of genomic

360 integration site on synthetic construct performance. We further harnessed S. boulardii's high

361 rates of homologous recombination to combinatorially assemble pathways for vitamin ( $\beta$ -

362 carotene) and drug (violacein) production. Finally, we measured S. boulardii's residence time in

363 germ-free and antibiotic-treated mice. Taken together, these results indicate that the decades of

364 work developing S. cerevisiae as a platform for biomanufacturing and synthetic biology can be

365 readily extended to S. boulardii. Coupled with the continued development of engineered

366 commensal and probiotic bacteria, in situ biomanufacturing by gut-resident microbes has the

367 potential to greatly reduce distributional complexity, improve site-specific delivery, and reduce 368 production costs for a wide array of biomolecules. 


\section{Acknowledgments}

371 The authors gratefully acknowledge the assistance of Dr. Susan Tonkonogy and Karen Flores in

372 performing germ-free mouse experiments. The authors thank Dr. Yong-Su Jin for kindly providing

373 S. boulardii knockout strains. They thank the lab of Dr. Albert Keung for assistance with flow

374 cytometry measurements. They thank the lab of Dr. Milad Abolhasani for assistance with HPLC

375 experiments. They also thank Dr. John E. Dueber for kindly sharing the MoClo-YTK Toolkit and

376 raw data. pLM494 was a gift from Bernd Müller-Röber (Addgene \#100539). LbCas12a was a gift

377 from Roubos Lab (Addgene \#101748). This work was supported by startup funds from North

378 Carolina State University's Chemical and Biomolecular Engineering Department. D.D. and I.S.A.

379 were supported by NCSU CBE startup funds. I.S.A. was also supported by the Ministry of Higher

380 Education - Oman.

381

382 Methods

383 Strains and Culture Media

384 Escherichia coli Top10, NEB $5 \alpha$ and NEB $10 \beta$ were used for plasmid construction and

385 maintenance. E. coli cells were grown in lysogeny broth (LB) (5g/L yeast extract, $10 \mathrm{~g} / \mathrm{L}$ tryptone,

$38610 \mathrm{~g} / \mathrm{L} \mathrm{NaCl})$ at $37{ }^{\circ} \mathrm{C}$ supplemented with ampicillin $(100 \mu \mathrm{g} / \mathrm{mL})$, kanamycin $(50 \mu \mathrm{g} / \mathrm{mL})$ or

387 chloramphenicol $(50 \mu \mathrm{g} / \mathrm{mL})$. Saccharomyces boulardii ATCC-MYA796 was used for antifungal

388 marker and origin of replication characterization. Saccharomyces boulardii $\triangle U R A 3$, provided by 
Yong-Su Jin, was used for auxotrophic marker, origin of replication, promoter, and terminator characterization, as well as genome editing and metabolic pathway assembly. Saccharomyces boulardii $\triangle T R P 1$ and $\triangle H I S 3$ strains from the Jin Lab were used for auxotrophic marker and origin

392 of replication characterization. Promoter and terminator characterization experiments were 393 conducted in synthetic media containing $0.67 \%(w / v)$ Yeast Nitrogen Base Without Amino Acids 394 (Sigma-Aldrich), 2\% Glucose (Fisher Scientific), 1.92g/L Yeast Synthetic Media Drop-Out Mix 395 without Uracil (Sigma-Aldrich). For auxotrophic marker characterization, synthetic media with 0.67\% (w/v) Yeast Nitrogen Base Without Amino Acids (Sigma-Aldrich), 2\% Glucose (Fisher

397 Scientific), 1.92\% Yeast Synthetic Media Drop-Out Mix without the appropriate nutrient 398 (histidine, tryptophan, or uracil) (Sigma-Aldrich) was used. Antifungal marker characterization experiments were conducted in yeast extract-peptone-dextrose medium (50 g/L YPD broth $\mu \mathrm{g} / \mathrm{mL}$ ) of geneticin (G418), zeocin, nourseothricin and hygromycin.

\section{Plasmid Construction \& Cloning}

404 All plasmids used in this work are listed in Supplementary File S1. A synthetic toolkit (MoClo-YTK) containing yeast parts were gifts from the Dueber Lab (Addgene \#:1000000061). Characterization

406 plasmids consisted of 8 parts: 2 connectors, promoter, coding sequence, terminator, yeast 407 marker, yeast origin, and E. coli marker and origin. Each of these plasmids were assembled 408 according to Deuber lab YTK protocols via Golden Gate cloning ${ }^{59}$. The Golden Gate reaction 409 mixture, for all plasmids except those containing $\beta$-carotene or violacein pathways, was prepared 410 as follows: $0.5 \mu \mathrm{L}$ of $40 \mathrm{nM}$ of each YTK plasmid (20 fmol), $0.5 \mu \mathrm{L}$ T7 Ligase (NEB) or $0.5 \mu \mathrm{L}$ T4 
411 Ligase (NEB), $1 \mu \mathrm{L}$ T4 Ligase Buffer (NEB) and $0.5 \mu \mathrm{L}$ Bsal (10000 U/mL, NEB) and water to bring

412 the final volume to $10 \mu \mathrm{L}$. The Golden Gate assembly protocol was performed on a thermocycler

413 with the following program: 30 cycles of digestion $\left(42{ }^{\circ} \mathrm{C}\right.$ for 2 minutes) and ligation $\left(16^{\circ} \mathrm{C}\right.$ for 5

414 minutes), followed by a final digestion $\left(60^{\circ} \mathrm{C}\right.$ for 10 minutes) and heat inactivation $\left(80^{\circ} \mathrm{C}\right.$ for 10

415 minutes).

\section{Yeast Competent Cells and Transformations}

418 We optimized the yeast competent cell preparation and transformation protocol from Gietz et.

419 al for Saccharomyces boulardii 60,61 . For the characterization experiments, we used frozen

420 competent cells, whereas for the genome editing experiments we used unfrozen competent cells

421 prepared on the same day ${ }^{60,61}$. To prepare competent cells, yeast colonies were inoculated into

$4221 \mathrm{~mL}$ YPD and incubated in a shaking incubator overnight at $37^{\circ} \mathrm{C}, 250 \mathrm{rpm}$. This culture was

423 diluted into fresh $50 \mathrm{~mL}$ YPD (approximately $\mathrm{OD}_{600}=0.25$ ), and grown for $6-8$ hours until $O D_{600}=1$.

424 The culture was centrifuged at $3000 \mathrm{xg}$ for $5 \mathrm{~min}$ at room temperature to pellet the cells. After

425 decanting the media, the pellet was resuspended in $25 \mathrm{~mL}$ of sterile water, and then pelleted at

$4263000 \mathrm{xg}$ for 5 minutes. This pellet was resuspended in $1 \mathrm{~mL}$ of sterile water. The cells were then

427 pelleted down at $10000 \mathrm{xg}$ for 1 minute and water was discarded. The cells were then

428 resuspended in $500 \mu \mathrm{L}$ of frozen competent cell solution consisting of $2.5 \%(\mathrm{v} / \mathrm{v})$ glycerol (Sigma-

429 Aldrich) and 5\% (v/v) DMSO (Thermo-Fisher). $50 \mu \mathrm{L}$ of this suspension was aliquoted into $1.5 \mathrm{~mL}$

430 microcentrifuge tubes and stored in $-80^{\circ} \mathrm{C}$. For transformations, frozen cells were thawed at $37^{\circ} \mathrm{C}$

431 for 1 minute. Then, the cells were pelleted by centrifugation at $3000 \mathrm{xg}$ for 2 minutes, after which

432 the supernatant was removed. Then, solutions were added in the following order; $260 \mu \mathrm{L} 50 \%$ 
433 PEG3350 (Fisher Scientific), $36 \mu \mathrm{L}$ 1M Lithium Acetate (Sigma-Aldrich), $50 \mu \mathrm{L}$ of $2 \mathrm{mg} / \mathrm{ml}$ single-

434 stranded salmon sperm DNA ((Invitrogen $\left.{ }^{\mathrm{TM}}, 15632011\right) 10 \mathrm{mg} / \mathrm{ml}$ double-stranded salmon sperm

435 DNA was diluted to $2 \mathrm{mg} / \mathrm{ml}$ and heat treated at $95^{\circ} \mathrm{C}$ for 5 minutes to denature the double-

436 stranded DNA), $0.1-10 \mu \mathrm{g}$ DNA and water to bring the final volume to $360 \mu \mathrm{L}$. The pellet was

437 resuspended in the transformation mix by gently mixing with a pipette tip. This transformation

438 mix was incubated at $42{ }^{\circ} \mathrm{C}$ for one hour. This mixture was then centrifuged at $3000 \times \mathrm{xg}$ for $1 \mathrm{~min}$

439 and the supernatant was discarded. The cell pellet was resuspended in $1 \mathrm{~mL}$ YPD by gently

440 pipetting up and down and this tube was incubated at $37{ }^{\circ} \mathrm{C}$ for one hour. Then, the cell

441 suspension was centrifuged for one minute at $3000 \mathrm{xg}$, resuspended in $25 \mu \mathrm{L}$ sterile water, and

442 plated on an appropriate growth media.

443

\section{S. boulardii Colony PCR}

445 Since S. boulardii has a thicker cell wall than S. cerevisiae, we developed a new method to 446 perform colony PCR on S. boulardii. Colonies were picked into $25 \mu \mathrm{L} 20 \mathrm{mM}$ sodium hydroxide

447 and incubated at $98^{\circ} \mathrm{C}$ for 80 minutes. Then we centrifuged these reactions at max speed (Mini

448 Microcentrifuge Sargent-Welch) for 10 minutes and started a $25 \mu \mathrm{L}$ PCR reaction with $2 \mu \mathrm{L}$ of this

449 reaction as a template. Otherwise, PCR reactions were performed according to the supplier's 450 instructions.

\section{Flow Cytometry}

453 Constructs were inoculated from -80C freezer stocks into $750 \mu \mathrm{L}$ of appropriate media in $96-$ 454 deep-well plates (VWR International) and incubated at $37{ }^{\circ} \mathrm{C}$ for 36 hours with shaking at 250 
$455 \mathrm{rpm}$. After 36 hours, the cultures were diluted 1:100 in $200 \mu \mathrm{L}$ of fresh media in 96-well-plates

456 (Costar) and were grown for $\sim 8-10$ hours at $37{ }^{\circ} \mathrm{C}$. Once the cultures reached $\mathrm{OD}_{600}$ values

457 between 0.1-0.4, flow cytometry was performed using a MACSQuant VYB (Miltenyi Biotec). Cells

458 containing empty vector were used as a control. The voltage of the channels were adjusted in

459 order to tune the gain of the detectors. The voltage values applied to FSC, SSC, Y1 (PE-A,

460 excitation:561, emission: 586/15 nm), V1 (CFP-VioBlue, excitation: $405 \mathrm{~nm}$, emission: 450/50 nm)

461 and B1 (GFP_FITC, excitation: $488 \mathrm{~nm}$, emission: 425/50 nm) channels/filters were 326V, 290V,

$462396 \mathrm{~V}, 324 \mathrm{~V}$ and $265 \mathrm{~V}$ respectively. Flow cytometry data analysis was conducted on the FlowJo

463 program (FlowJo LLC). The mean fluorescence values obtained from the flow cytometry for each

464 transcriptional construct was then normalized to background fluorescence (i.e. the cells with an

465 empty vector).

466

467 Mouse Experiments:

468 All mouse experiments were approved by the NC State University Institutional Animal Care and

469 Use Committee (IACUC).

470 Germfree Mice:

471 6-10 week old male and female germ free C57BL/6 mice were born at the NCSU Gnotobiotic

472 Animal Core and were mono-colonized by S. boulardii. 2 mice (males) were gavaged with $10^{6}$

473 colony forming units in $100 \mu \mathrm{L}$ PBS, 8 mice ( 6 females and 2 males) were gavaged with $10^{7}$ and 4

474 mice ( 2 females and 2 males) were gavaged with $10^{8} \mathrm{~S}$. boulardii cells on day 1 and fecal samples

475 were collected on days 2, 5 and 7. Fecal samples from mice housing the $10^{8}$ inoculum were 476 collected on day 30. 


\section{Conventional Mice:}

478 Six-week old female C57BL/6J mice were obtained from Jackson Labs and hosted at the NCSU 479 Biological Resources Facility (BRF) for 3-4 days before experiments. Mice were housed in groups 480 of three and their cages were changed before treatment with antibiotics and before treatment

481 with S. boulardii or water. All mice were treated with $1 \mathrm{mg} / \mathrm{ml}$ penicillin and $2 \mathrm{mg} / \mathrm{ml}$ streptomycin 482 in the drinking water for 4 days starting on day -3. On day 1, drinking water was either replenished 483 with antibiotic solution or changed to fresh water. Mice were then gavaged with $10^{8}$ CFU S. 484 boulardii or water once every 24 hours for 3 days. Fecal samples were collected every 24 hours 485 from day 1 to day 5.

\section{Stool Cultures.}

$487 \quad 1-2$ pieces of stool were collected in weighed $1.5 \mathrm{ml}$ centrifuge tubes and then weighed again to 488 determine fecal mass. Fecal matter was then resuspended in $1 \mathrm{~mL}$ PBS per $100 \mathrm{mg}$ feces. Fecal 489 suspensions from mono-colonized mice were placed on solid YPD media, while fecal suspensions 490 from conventional mice treated with antibiotics were plated on YPD media containing $50 \mu \mathrm{g} / \mathrm{ml}$ 491 nourseothricin and $0.25 \mu \mathrm{g} / \mathrm{ml}$ streptomycin. Plates were sealed with parafilm and incubated at $49237^{\circ} \mathrm{C}$ for $2-3$ days.

\section{Genome Editing:}

495 Integration plasmids contained URA3 to aid selection and contained homology arms between 496350 to 900 base-pairs in length as shown in Supplementary Figure S7. For DNA homologous 497 recombination without DSB (dsDNA integration), $2 \mu \mathrm{g}$ of linear donor DNA was transformed to $49810^{7}$ S. boulardii (S. boulardii $\triangle U R A 3$ ) competent cells prepared on the same day. For SpCas9, $2 \mu \mathrm{g}$ 
499 of Linear donor DNA and $1.5 \mu \mathrm{g}$ of gRNA plasmids were transformed to $10^{7}$ freshly prepared

500 chemical competent S. boulardii $\triangle U R A 3$ cells containing SpCas9 plasmid. For LbCas12a, $2 \mu \mathrm{g}$ of

501 Linear donor DNA and $1.5 \mu \mathrm{g}$ of gRNA plasmids were transformed to $10^{7}$ freshly prepared

502 chemical competent S. boulardii $\triangle U R A 3$ cells LbCas12a plasmid.

503

504 Overnight cultures of $S$. boulardii $\triangle U R A 3$ were grown in YPD only for Homologous

505 Recombination, S. boulardii $\triangle U R A 3$ containing the SpCas9 plasmid were grown in YPD +100

$506 \mu \mathrm{g} / \mathrm{mL}$ nourseothricin, and S. boulardii $\triangle U R A 3$ containing the LbCas12a plasmid were grown in

507 YPD $+200 \mu \mathrm{g} / \mathrm{mL} \mathrm{G418}$ were subinoculated to prewarmed YPD media with or without antifungals

508 at an $\mathrm{OD}_{600}$ of 0.25 and incubated at $37^{\circ} \mathrm{C}$ with shaking at $250 \mathrm{rpm}$ until cultures reached an

$509 \mathrm{OD}_{600}$ of $0.9-1.0$. Cultures were then centrifuged at $3000 \mathrm{xg}$ for $5 \mathrm{~min}$. Cells were then washed

510 twice with 0.5 and 0.1 volumes of sterile DI water at room temperature at $3000 x g$ for $5 \mathrm{~min}$. The

511 cells then were transformed according to the protocol described above, with the exception that

512 heat shocked cells were spun down, resuspended in YPD, and incubated for 3 hours at $37^{\circ} \mathrm{C}$.

513 Transformed cells were then spread on Yeast Complete Synthetic Media (CSM) without uracil

514 plates with or without antibiotics. Plates were incubated at $37^{\circ} \mathrm{C}$ for $2-4$ days. Integration was

515 verified with colony PCR.

517 Combinatorial Pathway Assembly

518 Prior to library assembly, promoter, gene, and terminator part plasmids were assembled into

519 'entry vector' plasmids by BsmBI assembly. These plasmids had Bsal overhangs and follow the

520 'Yeast golden gate toolkit' standard [deuber gg]. Backbones were assembled for subcloning of 
521 the transcriptional units. These backbones housed the appropriate homology arms for later final

522 assembly of transcriptional units into a full pathway. Bsal assembly of transcriptional units into

523 the matching backbone was completed by combining $10 \mathrm{fmol}$ of each promoter part plasmid (to

524 a total of $100 \mathrm{fmol}$ ) with $100 \mathrm{fmol}$ of gene, terminator, and backbone plasmids. Reaction mixtures

525 included $0.5 \mu \mathrm{L}$ of Bsal-HF v2, $0.5 \mu \mathrm{L}$ of T7 DNA Ligase, and $1 \mu \mathrm{L}$ of T4 DNA ligase buffer (NEB),

526 and underwent 50 cycles of golden gate assembly ( 2 minutes at $42^{\circ} \mathrm{C}, 5$ minutes at $\left.16^{\circ} \mathrm{C}\right)$, before

527 a single 30 minute final digestion at $60{ }^{\circ} \mathrm{C}$, and 10 minutes of heat inactivation at $80{ }^{\circ} \mathrm{C}$.

528 Subsequently, we have noticed that final digestion steps of 5 minutes at $42{ }^{\circ} \mathrm{C}$ are more optimal

529 for Bsal assembly ${ }^{59}$. Transcriptional units were amplified directly from the golden gate mixture

530 using $1 \mu \mathrm{L}$ of reaction mixture as template in $50 \mu \mathrm{L}$ Q5 Hot-Start Master Mix reactions (NEB). PCR

531 reactions were performed according to manufacturer's instructions and cleaned up using clean

532 and concentrate kits (D4004-Zymo Research). $600 \mathrm{nM}$ of each amplified transcriptional unit

533 library was transformed using the lithium acetate protocol described above. Cells were recovered

534 for 1 hour in YPD prior to dividing the culture into 5 aliquots of $200 \mu \mathrm{L}$ and plating on yeast

535 nitrogen base plus amino acids without uracil (Sigma).

\section{Pathway Sequencing.}

538 Random colonies were collected in 1.5 centrifuge tubes and resuspended in $1 \mathrm{ml} \mathrm{of} 10 \mathrm{mg} / \mathrm{ml}$

539 Lysing Enzymes from Trichoderma harzianum (L1412 Sigma). The cells were incubated in the

540 lysing enzyme at $37^{\circ} \mathrm{C}$ for 3 hours and then the ZymoPURE Plasmid Miniprep (D4208T) was used

541 to miniprep the plasmids. The Lysing Enzymes from Trichoderma harzianum help digest $S$.

542 boulardii's wall and produce protoplasts that are easy to miniprep. $2 \mu l$ of the miniprep product 
543 was then used as a template to amplify the assembled region with primers complementary to

544 the pathway genes or terminators, as the ordering of these was common to all constructs. The

545 PCR product was then sent for Sanger sequencing (Genewiz, NJ,USA) to verify the promoters

546 assigned for each gene.

547

548 Antifungal Minimum Inhibitory Concentration tests in S. boulardii:

5493 biological replicates of S. boulardii (ATCC-MYA796) were grown overnight in YPD media.

550 Overnight cultures were sub-inoculated into YPD media with different antifungal concentrations

551 ranging from $0 \mu \mathrm{g} / \mathrm{ml}$ to $200 \mu \mathrm{g} / \mathrm{ml}$ in a 96 well plate at starting OD $_{600} 0.02$ and grown for 36

552 hours in a plate reader (BioTek Synergy ${ }^{\top M}$ H1, Shake Mode: Double Orbital, Orbital Frequency:

553 continuous shake 365 cpm, Interval: 10 minutes). Geneticin (G418), Nourseothricin, Zeocin,

554 Hygromycin B were used in this study.

555

556 Extraction of $\beta$-carotene from S. boulardii cultures.

5573 replicates of each $\beta$-carotene producing $S$. boulardii strain were inoculated into $1 \mathrm{~mL}$ CSM

558 without uracil ( $2.5 \%$ glucose) and and grown overnight in a shaking incubator at $250 \mathrm{rpm}$ at $37^{\circ} \mathrm{C}$.

559 Overnight cultures were then subinoculated (1:100) into $30 \mathrm{~mL} \mathrm{CSM}$ without uracil (2.5\% glucose)

560 and grown for 3 days in a rotary incubator at $250 \mathrm{rpm}$ at $37^{\circ} \mathrm{C}$. After 3 days, $2^{*} 10^{9}$ cells were

561 collected by centrifuging at $4{ }^{\circ} \mathrm{C}$ at $5000 \mathrm{~g}$ for 5 minutes. The media was discarded and the cells

562 were washed with $10 \mathrm{~mL}$ water and were centrifuged at $4{ }^{\circ} \mathrm{C}$ at $5000 \mathrm{~g}$ for another 5 minutes.

563 After the water was discarded, the cells were resuspended in $500 \mu \mathrm{L}$ water and transferred into

564 ZR Bashing Bead Tubes (Zymo Research, CA). The ZR Bashing tubes were centrifuged for 1 minute 
565 at $8000 \mathrm{~g}$ to discard the water. $500 \mu \mathrm{L}$ acetone was then added into the bashing tubes. The tubes

566 containing the cells, acetone, and lysis beads then transferred to a homogenizer (TissueLyser,

567 Qiagen, Germany) for physical lysis (Lysis duration: 10 minutes, frequency: 30/s). The cells and

568 the lysates then centrifuged at $16000 \mathrm{~g}$ for 5 minutes at $4{ }^{\circ} \mathrm{C}$. The supernatants (acetone lysate)

569 were transferred to $1.5 \mathrm{~mL}$ black microcentrifuge tubes. Fresh acetone was then added to the

570 bashing tubes, and lysis steps were repeated 2 more times, at which point the cell pellets were

571 completely white. $\beta$-carotene extracts were dried by speed vac (aqueous solvent, $30{ }^{\circ} \mathrm{C}$ for 2

572 hours). Dried $\beta$-carotene pellets were reconstituted in $500 \mu \mathrm{L}$ acetone and stored at $-20^{\circ} \mathrm{C}$ in the

573 dark.

574

\section{Detection of $\beta$-carotene by HPLC.}

$5765 \mu \mathrm{L}$ of each sample was processed in an Agilent 1260 Infinity high performance liquid-

577 chromatography (HPLC) system (Agilent Technologies, CA, USA). 0.002 mM naphthalene solution

578 was used as internal control and pure $\beta$-carotene (Thermo Fisher, Waltham, MA) at varying

579 concentrations $(\mathrm{mg} / \mathrm{mL}) 0.00625,0.0125,0.025,0.05,0.0625,0.125,0.25,0.5,0.625,1.25,2.5$,

580 5) as external control. The channels used for internal control and $\beta$-carotene detection were 275

$581 \mathrm{~nm}$ and $453 \mathrm{~nm}$, respectively. The column used in the system was an Agilent HC-C28 Reversed

582 Phase column (4.6 x $250 \mathrm{~mm}, 50 \mu \mathrm{L})$ (Agilent Technologies, CA, USA). The composition of the

583 mobile phase was acetonitrile:methanol:isopropanol (v/v/v: 85/10/5) The flow rate for the

584 mobile phase was $3 \mathrm{~mL} / \mathrm{min}$. 
587 1. Tiago, F. C. P. et al. Adhesion to the yeast cell surface as a mechanism for trapping pathogenic bacteria by Saccharomyces probiotics. J. Med. Microbiol. 61, 1194-1207 (2012).

2. Khatri, I., Tomar, R., Ganesan, K., Prasad, G. S. \& Subramanian, S. Complete genome sequence and comparative genomics of the probiotic yeast Saccharomyces boulardii. Sci. Rep. 7, 371 (2017).

3. O'Toole, P. W., Marchesi, J. R. \& Hill, C. Next-generation probiotics: the spectrum from probiotics to live biotherapeutics. Nat Microbiol 2, 17057 (2017).

4. Mimee, M., Citorik, R. J. \& Lu, T. K. Microbiome therapeutics - Advances and challenges. Adv. Drug Deliv. Rev. 105, 44-54 (2016).

5. Pedrolli, D. B. et al. Engineering Microbial Living Therapeutics: The Synthetic Biology Toolbox. Trends Biotechnol. 37, 100-115 (2019).

6. Ozdemir, T., Fedorec, A. J. H., Danino, T. \& Barnes, C. P. Synthetic Biology and Engineered Live Biotherapeutics: Toward Increasing System Complexity. Cell Syst 7, 5-16 (2018).

600 7. Ferreiro, A., Crook, N., Gasparrini, A. J. \& Dantas, G. Multiscale Evolutionary Dynamics of Host-Associated Microbiomes. Cell 172, 1216-1227 (2018). in mammalian hosts. Nature 553, 86-90 (2018).

9. Riglar, D. T. et al. Engineered bacteria can function in the mammalian gut long-term as live diagnostics of inflammation. Nat. Biotechnol. 35, 653-658 (2017). 
mammalian gut. Proc. Natl. Acad. Sci. U. S. A. 111, 4838-4843 (2014).

11. Shaw, W. M. et al. Engineering a Model Cell for Rational Tuning of GPCR Signaling. Cell 177, 782-796.e27 (2019).

12. Rugbjerg, P. \& Sommer, M. O. A. Overcoming genetic heterogeneity in industrial fermentations. Nat. Biotechnol. (2019) doi:10.1038/s41587-019-0171-6. 504 (2019).

14. Sender, R., Fuchs, S. \& Milo, R. Revised Estimates for the Number of Human and Bacteria Cells in the Body. PLoS Biol. 14, e1002533 (2016).

15. Crook, N. et al. Adaptive Strategies of the Candidate Probiotic E. coli Nissle in the Mammalian Gut. Cell Host Microbe 25, 499-512.e8 (2019).

16. Isabella, V. M. et al. Development of a synthetic live bacterial therapeutic for the human metabolic disease phenylketonuria. Nat. Biotechnol. 36, 857-864 (2018).

17. Kurtz, C. B. et al. An engineered E. coli Nissle improves hyperammonemia and survival in mice and shows dose-dependent exposure in healthy humans. Sci. Transl. Med. 11, (2019). chemoprevention. Nat Biomed Eng 2, 27-37 (2018).

19. Hwang, I. Y. et al. Engineered probiotic Escherichia coli can eliminate and prevent 
21. Sweere, J. M. et al. Bacteriophage trigger antiviral immunity and prevent clearance of bacterial infection. Science 363, (2019).

631 22. Brunk, E. et al. Characterizing posttranslational modifications in prokaryotic metabolism using a multiscale workflow. Proc. Natl. Acad. Sci. U. S. A. 115, 11096-11101 (2018).

23. Jiang, T. T. et al. Commensal Fungi Recapitulate the Protective Benefits of Intestinal Bacteria. Cell Host Microbe 22, 809-816.e4 (2017).

24. Huseyin, C. E., O’Toole, P. W., Cotter, P. D. \& Scanlan, P. D. Forgotten fungi-the gut mycobiome in human health and disease. FEMS Microbiol. Rev. 41, 479-511 (2017).

637 25. Wheeler, M. L. et al. Immunological Consequences of Intestinal Fungal Dysbiosis. Cell Host Microbe 19, 865-873 (2016).

26. Hamad, I. et al. Culturomics and Amplicon-based Metagenomic Approaches for the Study of Fungal Population in Human Gut Microbiota. Sci. Rep. 7, 16788 (2017). interactions and role in gastrointestinal diseases. Nat. Rev. Gastroenterol. Hepatol. 16,

644 28. Shao, T.-Y. et al. Commensal Candida albicans Positively Calibrates Systemic Th17

645 Immunological Responses. Cell Host Microbe 25, 404-417.e6 (2019).

646 29. Tso, G. H. W. et al. Experimental evolution of a fungal pathogen into a gut symbiont. Science 362, 589-595 (2018).

648 30. Hotel, A. C. P. \& Cordoba, A. Health and nutritional properties of probiotics in food including powder milk with live lactic acid bacteria. Prevention 5, 1-10 (2001). 
evaluated by sequence analysis of the D1/D2 domain of 26S rDNA, the ITS1-5.8S rDNA-ITS2 $571(2003)$.

32. Hudson, L. E. et al. Characterization of the Probiotic Yeast Saccharomyces boulardii in the Healthy Mucosal Immune System. PLoS One 11, e0153351 (2016).

33. Liu, J.-J. et al. Metabolic Engineering of Probiotic Saccharomyces boulardii. Appl. Environ. Microbiol. 82, 2280-2287 (2016).

34. Fietto, J. L. R. et al. Molecular and physiological comparisons between Saccharomyces cerevisiae and Saccharomyces boulardii. Can. J. Microbiol. 50, 615-621 (2004). phagocytic system of gnotobiotic mice : immunomodulation by probiotic. J. Appl. Microbiol. 89, 404-414 (2000).

36. Palma, M. L., Garcia-Bates, T. M., Martins, F. S. \& Douradinha, B. Genetically engineered (2019).

37. Michael, S. et al. Quantitative phenotyping of inflammatory bowel disease in the IL-10deficient mouse by use of noninvasive magnetic resonance imaging. Inflamm. Bowel Dis.

670 38. Lee, M. E., DeLoache, W. C., Cervantes, B. \& Dueber, J. E. A Highly Characterized Yeast Toolkit for Modular, Multipart Assembly. ACS Synth. Biol. 4, 975-986 (2015).

672 39. Karim, A. S., Curran, K. A. \& Alper, H. S. Characterization of plasmid burden and copy 
number in Saccharomyces cerevisiae for optimization of metabolic engineering applications. FEMS Yeast Res. 13, 107-116 (2013).

675

40. Ghim, C.-M., Lee, S. K., Takayama, S. \& Mitchell, R. J. The art of reporter proteins in science: past, present and future applications. BMB Rep. 43, 451-460 (2010).

41. Xiao, Y., Bowen, C. H., Liu, D. \& Zhang, F. Exploiting nongenetic cell-to-cell variation for enhanced biosynthesis. Nat. Chem. Biol. 12, 339-344 (2016).

42. Mundt, M., Anders, A., Murray, S. M. \& Sourjik, V. A System for Gene Expression Noise Control in Yeast. ACS Synth. Biol. 7, 2618-2626 (2018).

43. Yuan, T. et al. Construction, characterization and application of a genome-wide promoter library in Saccharomyces cerevisiae. Frontiers of Chemical Science and Engineering 11, 107116 (2017).

44. Partow, S., Siewers, V., Bjørn, S., Nielsen, J. \& Maury, J. Characterization of different promoters for designing a new expression vector in Saccharomyces cerevisiae. Yeast 27, 955-964 (2010).

45. Datta, S., Annapure, U. S. \& Timson, D. J. Different specificities of two aldehyde dehydrogenases from Saccharomyces cerevisiae var. boulardii. Biosci. Rep. 37, (2017).

46. Curran, K. A., Karim, A. S., Gupta, A. \& Alper, H. S. Use of expression-enhancing terminators in Saccharomyces cerevisiae to increase mRNA half-life and improve gene expression control for metabolic engineering applications. Metab. Eng. 19, 88-97 (2013). 
695

696

697

698

699

700

701

702

703

704

705

706

707

708

709

710

711

712

713

714

715

716

48. Yamanishi, M. et al. A genome-wide activity assessment of terminator regions in Saccharomyces cerevisiae provides a "terminatome" toolbox. ACS Synth. Biol. 2, 337-347 (2013).

49. Struhl, K. \& Segal, E. Determinants of nucleosome positioning. Nat. Struct. Mol. Biol. 20, 267-273 (2013).

50. Raschmanová, H., Weninger, A., Glieder, A., Kovar, K. \& Vogl, T. Implementing CRISPR-Cas technologies in conventional and non-conventional yeasts: Current state and future prospects. Biotechnol. Adv. 36, 641-665 (2018).

51. Yuan, J. \& Ching, C. B. Combinatorial assembly of large biochemical pathways into yeast chromosomes for improved production of value-added compounds. ACS Synth. Biol. 4, 2331 (2015).

52. Lee, M. E., Aswani, A., Han, A. S., Tomlin, C. J. \& Dueber, J. E. Expression-level optimization of a multi-enzyme pathway in the absence of a high-throughput assay. Nucleic Acids Res. 41, 10668-10678 (2013).

53. Wingler, L. M. \& Cornish, V. W. Reiterative Recombination for the in vivo assembly of libraries of multigene pathways. Proc. Natl. Acad. Sci. U. S. A. 108, 15135-15140 (2011).

54. Verwaal, R. et al. High-level production of beta-carotene in Saccharomyces cerevisiae by successive transformation with carotenogenic genes from Xanthophyllomyces dendrorhous. Appl. Environ. Microbiol. 73, 4342-4350 (2007).

55. Cunningham, F. X. \& Gantt, E. One ring or two? Determination of ring number in carotenoids by lycopene $\varepsilon$-cyclases. Proc. Natl. Acad. Sci. U. S. A. 98, 2905-2910 (2001).

56. Naseri, G., Behrend, J., Rieper, L. \& Mueller-Roeber, B. COMPASS for rapid combinatorial 
717 optimization of biochemical pathways based on artificial transcription factors. Nat.

718 Commun. 10, 2615 (2019).

719 57. Kennedy, E. A., King, K. Y. \& Baldridge, M. T. Mouse Microbiota Models: Comparing Germ-

720 Free Mice and Antibiotics Treatment as Tools for Modifying Gut Bacteria. Front. Physiol. 9,

$721 \quad 1534(2018)$

722 58. Barc, M.-C. et al. Molecular analysis of the digestive microbiota in a gnotobiotic mouse

723 model during antibiotic treatment: Influence of Saccharomyces boulardii. Anaerobe 14,

$724 \quad 229-233(2008)$.

725 59. Potapov, V. et al. Comprehensive Profiling of Four Base Overhang Ligation Fidelity by T4

726 DNA Ligase and Application to DNA Assembly. ACS Synth. Biol. 7, 2665-2674 (2018).

727 60. Gietz, R. D., Daniel Gietz, R. \& Schiestl, R. H. High-efficiency yeast transformation using the 728 LiAc/SS carrier DNA/PEG method. Nature Protocols vol. 2 31-34 (2007).

729 61. Gietz, R. D., Daniel Gietz, R. \& Schiestl, R. H. Quick and easy yeast transformation using the 730 LiAc/SS carrier DNA/PEG method. Nature Protocols vol. 2 35-37 (2007). 


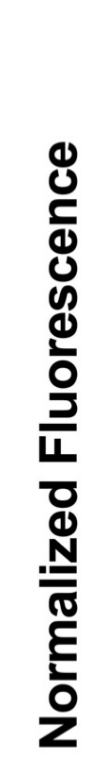

1000

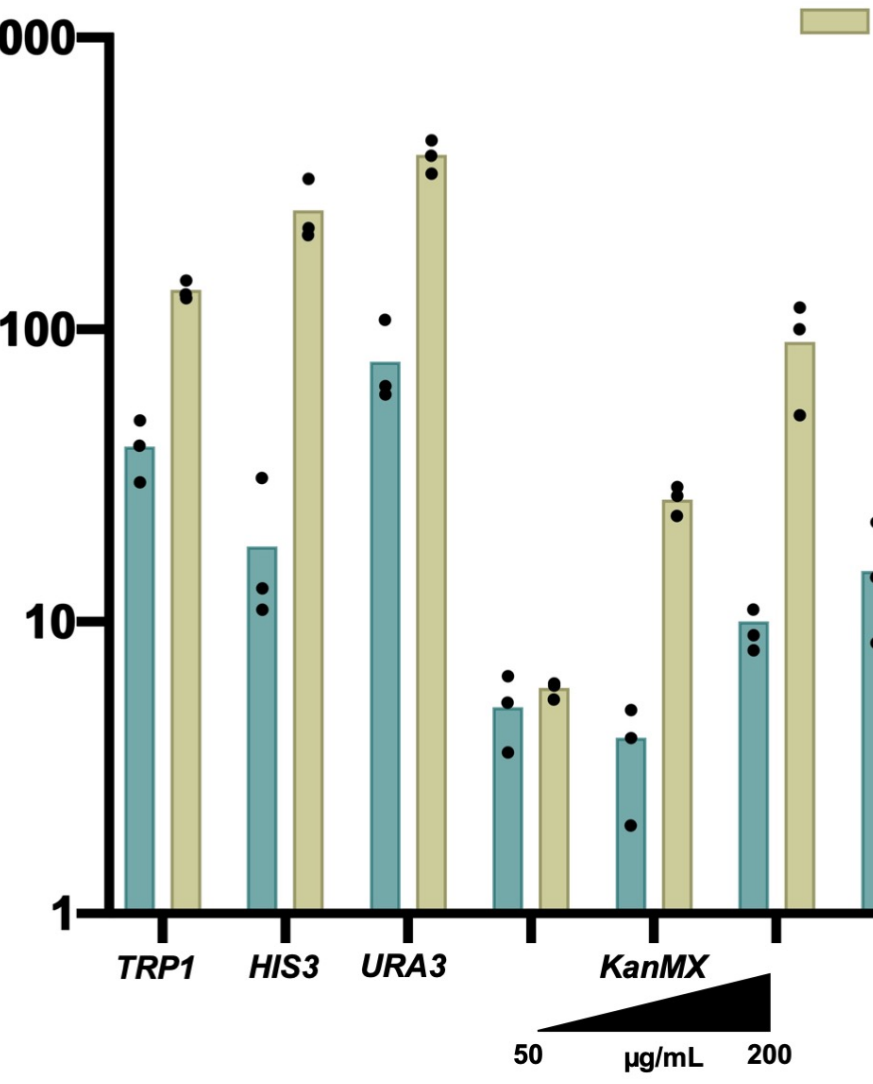

$\mathrm{b}$

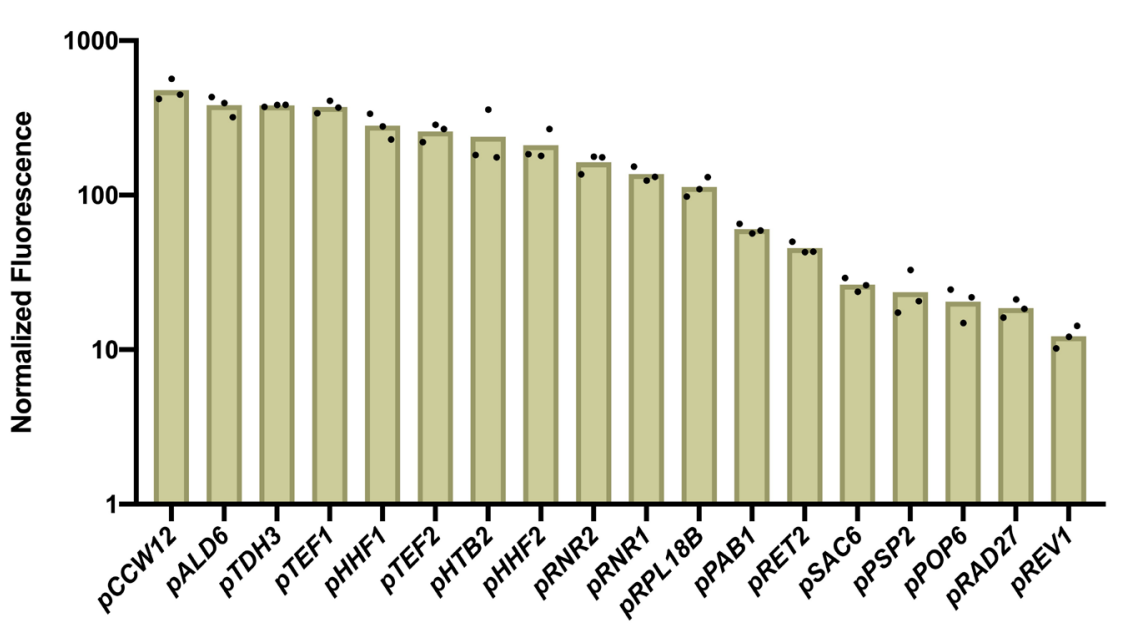

C

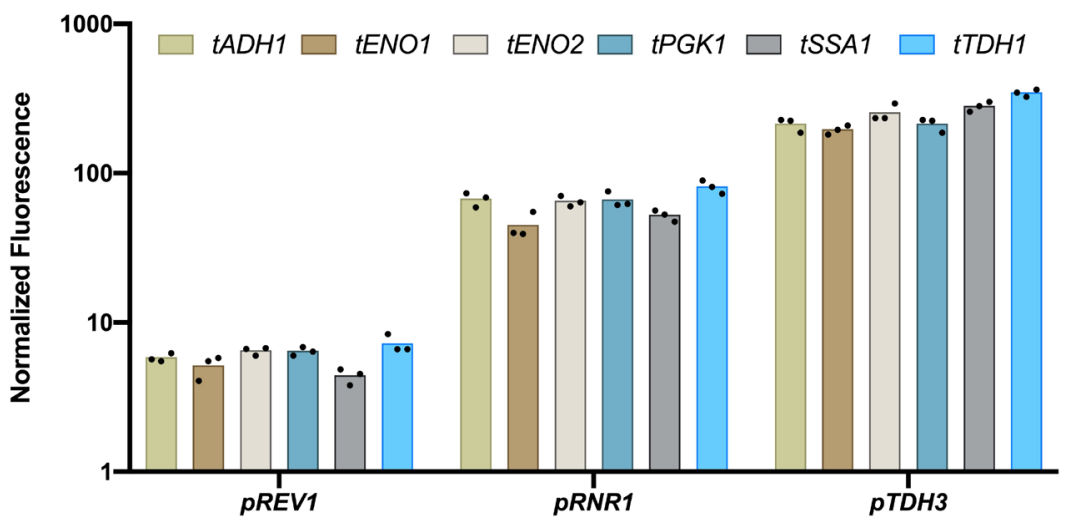

Figure 1. Characterization of $S$. boulardii (a) markers and origins of replication, (b) promoters, and (c) terminators in vitro. a) 3 auxotrophic (URA3, HIS3, TRP1) and 4 antifungal (KanMX, ZeoR, NatR, HygR) markers were cloned in plasmids containing the TDH3 promoter, mRuby2 fluorescent protein and TDH1 terminator. Centromeric (CEN6/ARS4) and episomal (2 $\mu$ ) origins were cloned in combination with the 7 selective markers. Plasmids with antifungal markers were tested in 3 different antifungal concentrations (50, $100 \mathrm{and} 200 \mu \mathrm{g} / \mathrm{mL}) . \mathbf{b}) 18 \mathrm{constitutive} \mathrm{promoters} \mathrm{were}$ cloned 5' of the mRuby2 fluorescent protein and the TDH1 terminator. c) 5 terminators were cloned 3' of 3 different promoters at different strength ( $p$ TDH3-strong, $p R N R 1$-medium and $p R E V 1$ weak) and the mRuby2 fluorescent protein. Absolute fluorescence values were obtained via flow cytometry for 3 biological replicates $(n=3)$. Absolute fluorescence values were normalized to fluorescent values of cells harboring the spacer plasmid. The height of the bars represents the normalized mean fluorescent value of 3 biological replicates and the black dots show the normalized mean of each biological replicate. 

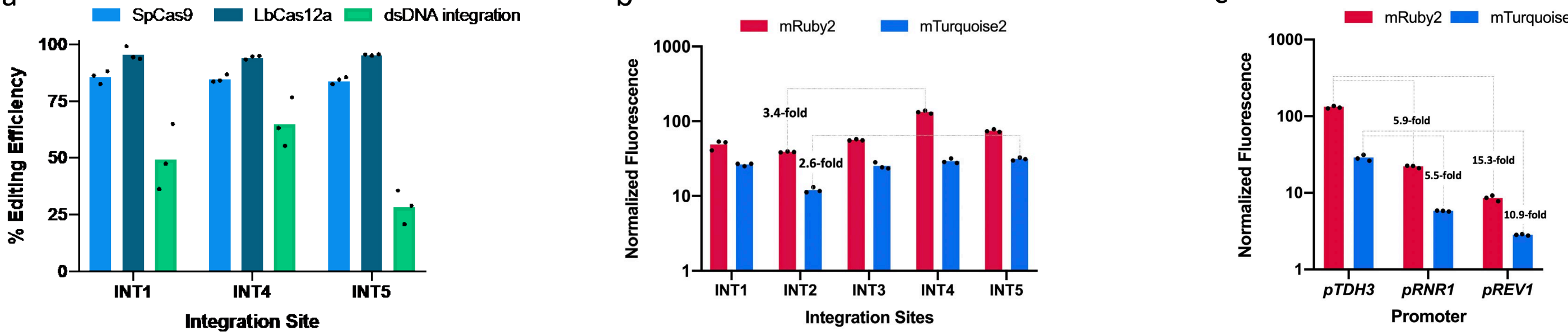

Figure 2. S. boulardii genomic location impacts gene expression, and comparison of genome editing techniques. a. Editing efficiency at 3 different loci using 3 different methods: CRISPR/SpCas9 (SpCas9), CRISPR/LbCas12a (LbCas12a) and un-assisted linear dsDNA integration (dsDNA integration). 3 different guides were used for both SpCas9 and LbCas12a for each site. Each dot represents one guide. 3 replicate cultures were transformed with the DNA repair template to measure the efficiency of genome editing in $S b$ via dsDNA integration. Table $\mathrm{S} 1$ shows the number of colonies screen for each method. b. Effect of chromosomal locus on the expression of the fluorescent genes mRuby 2 and mTurqouise2. The dotted lines show the fold change between the expression of mRuby 2 and mTurquoise 2 between the highest and lowest expression among all the integration sites. Each dot represents one biological replicate. c. The effects of promoter (strong, medium, or weak) on gene expression at the same chromosomal locus. The dotted lines show the fold change between the expression of $m R$ Ruby 2 and mTurquoise 2 using different promoters. Each dot represents one biological replicate. 
b

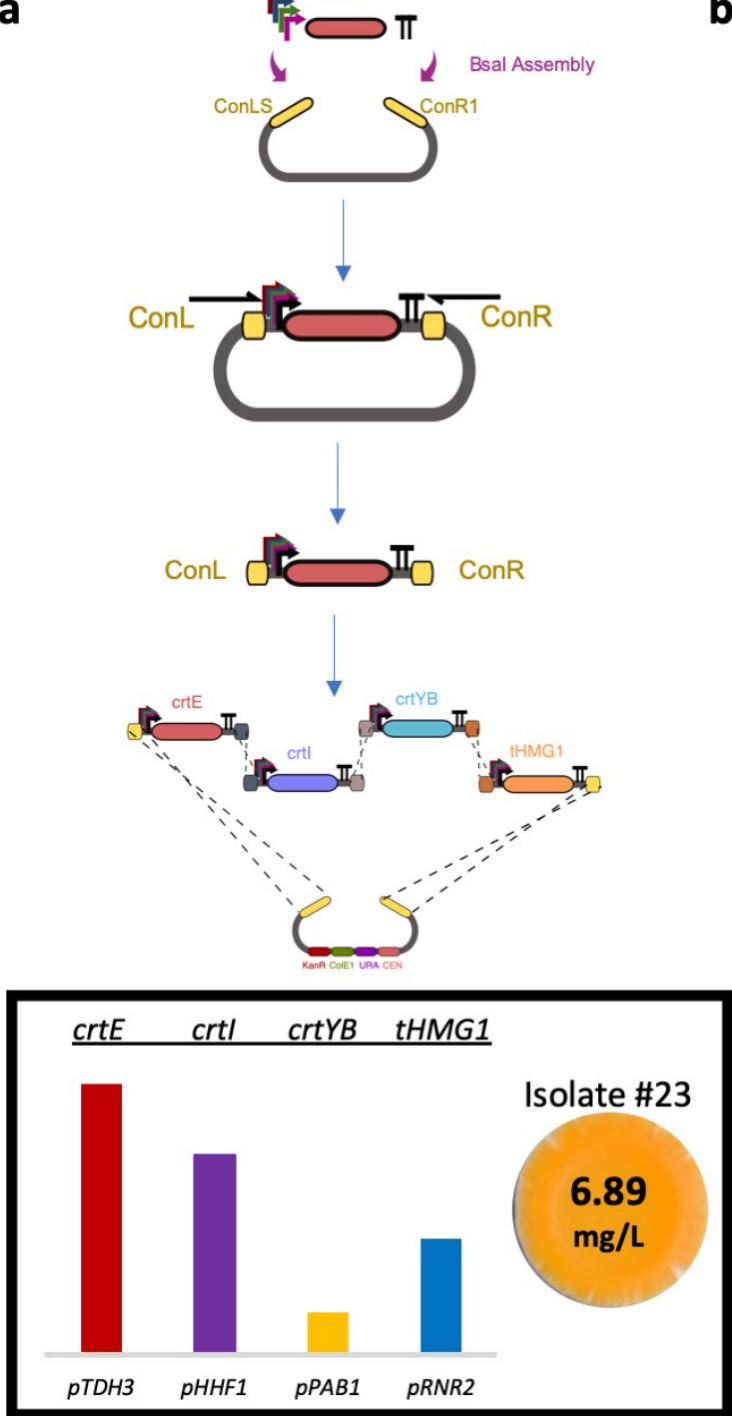

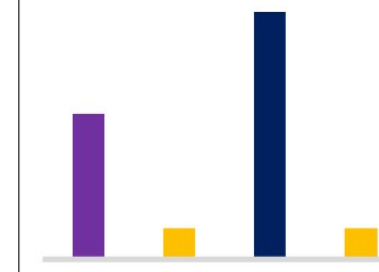

PHHF1 PPAB1 PCCW12 PPAB

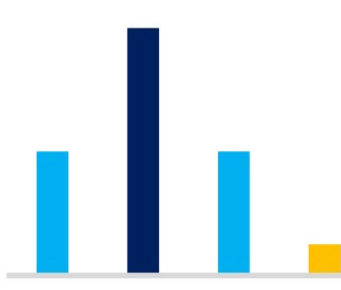

PHTB2 pCCW12 PHTB2 PPAB1

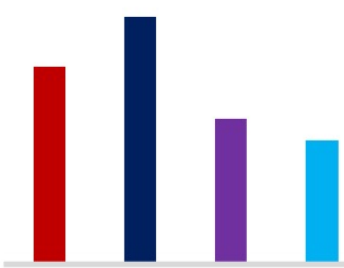

PTDH3 рCCW12 PHHF1

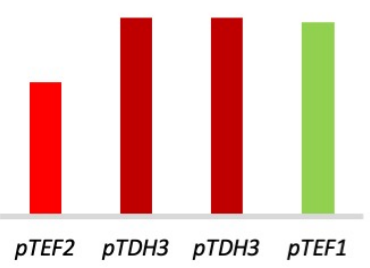

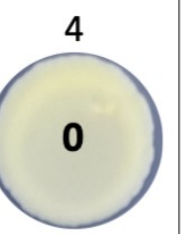

10

0.75

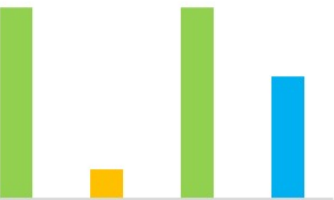

PTEF1 PPAB1 PTEF1 PHTB2

15

0.63

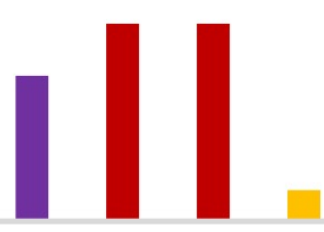

PHHF1 PTDH3 PTDH3 PPAB1

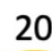

0.23

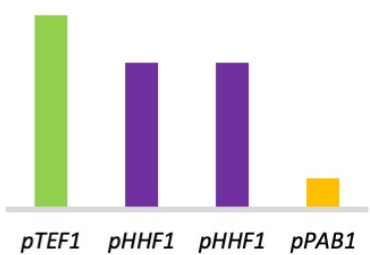

1

0

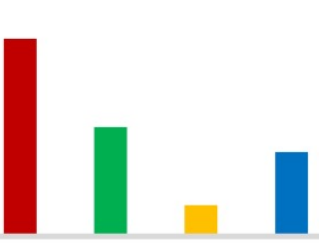

11

0.78

16

0.78

22

0.37
PTDH3 PHHF2 PPAB1 PRNR2

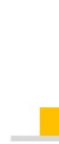

PPAB1

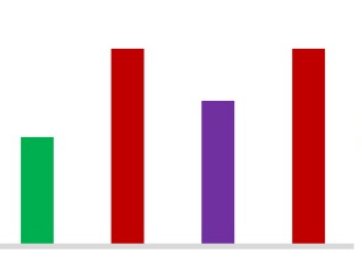

PHHF2 2 PTDH3

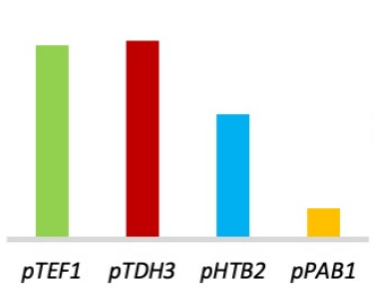

0

0

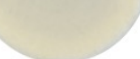

17

0.20

6

7.27

PTEF1 PTDH3

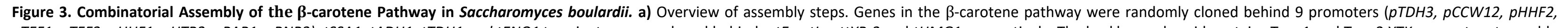

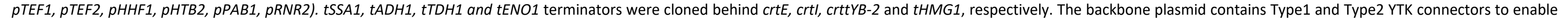

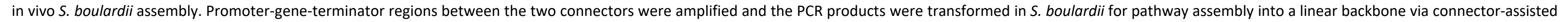

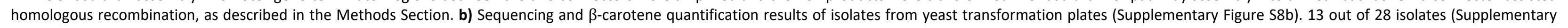

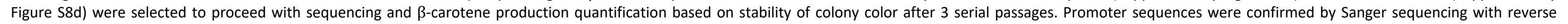

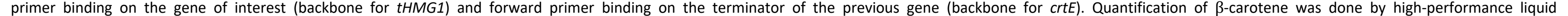

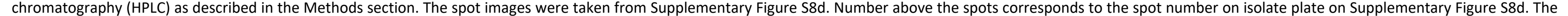

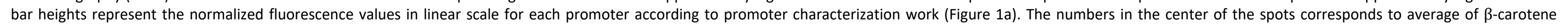
produced by 3 biological replicate of each isolate in one liter of saturated yeast culture. 
$a$

Germfree mice

b

Conventional

Mice

C

Conventional

Mice

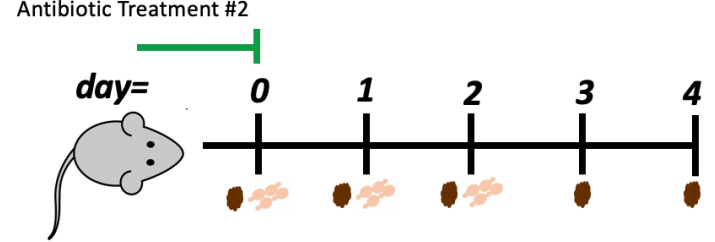

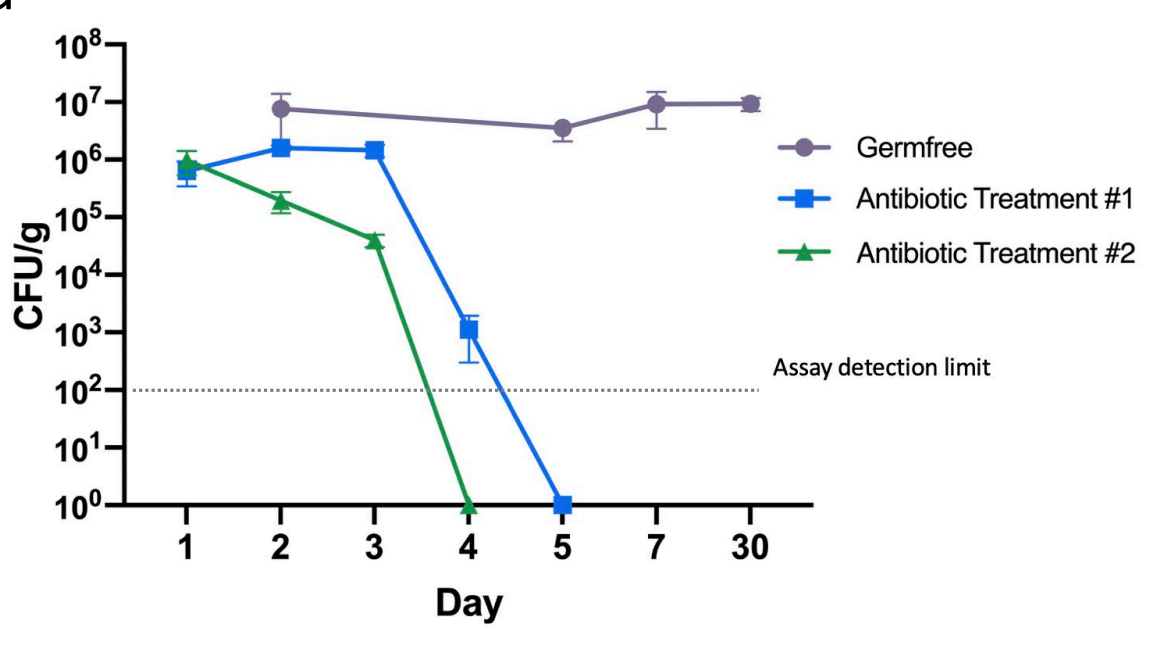

Figure 4. Microbial competition reduces $S$. boulardii residence time in mice. a. Germfree mouse model gavaged with $S$. boulardii on day 0. b Conventional mouse model treated with $1 \mathrm{mg} / \mathrm{ml}$ penicillin and $2 \mathrm{mg} / \mathrm{ml}$ streptomycin in the drinking water for 4 days prior to 3 S. $b:: N a t R$ treatments every 24 hours (antibiotics were replenished every 4 days.) c. Conventional mouse model treated with $1 \mathrm{mg} / \mathrm{ml}$ penicillin and $2 \mathrm{mg} / \mathrm{ml}$ streptomycin in the drinking water for 4 days prior to 3 S. $b::$ NatR treatments every 24 hours (antibiotic treatment was stopped on day 0.) d. Fecal carriage of $S . b$ in the three mice models. Fecal samples were collected as shown in the timelines and plated on YPD media for the gnotobiotic model and YPD containing antibiotics for the conventional mice models. The dashed line shows the detection limit of our assay. Error bars indicate the standard deviation observed among 4 germ free mice, 3 conventional mice treated with antibiotic treatment $\# 1$, and 3 conventional mice treated with antibiotic treatment \#2. In all mouse models, feces were collected prior to Sb gavage during days when both occurred. 

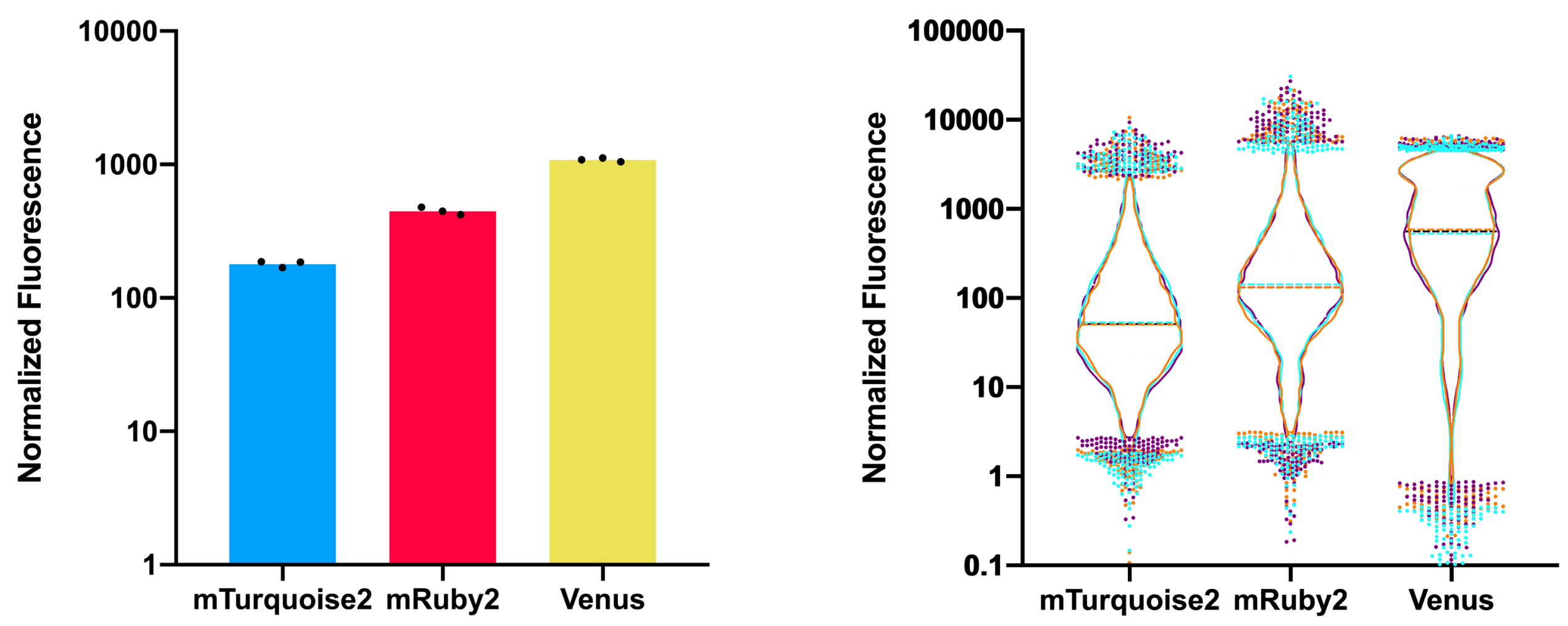

Figure S1. Characterization of Fluorescent Genes. a) 3 fluorescent genes (mTurquoise2, mRuby2 and Venus) were cloned behind TDH3 promoter and in front of TDH1 terminator. The height of the bars represent the mean fluorescent value of 3 biological replicates $(n=3)$. The black dots show the normalized mean of each biological replicate. $\mathbf{b})$ Violin plots of flow cytometry data for 3 fluorescent proteins. Cyan, orange and purple represent first, second and third biological replicates, respectively. The dots represent the highest and lowest 2 percent of the cell population. Each flow cytometry run consists of 10000 events. 
a

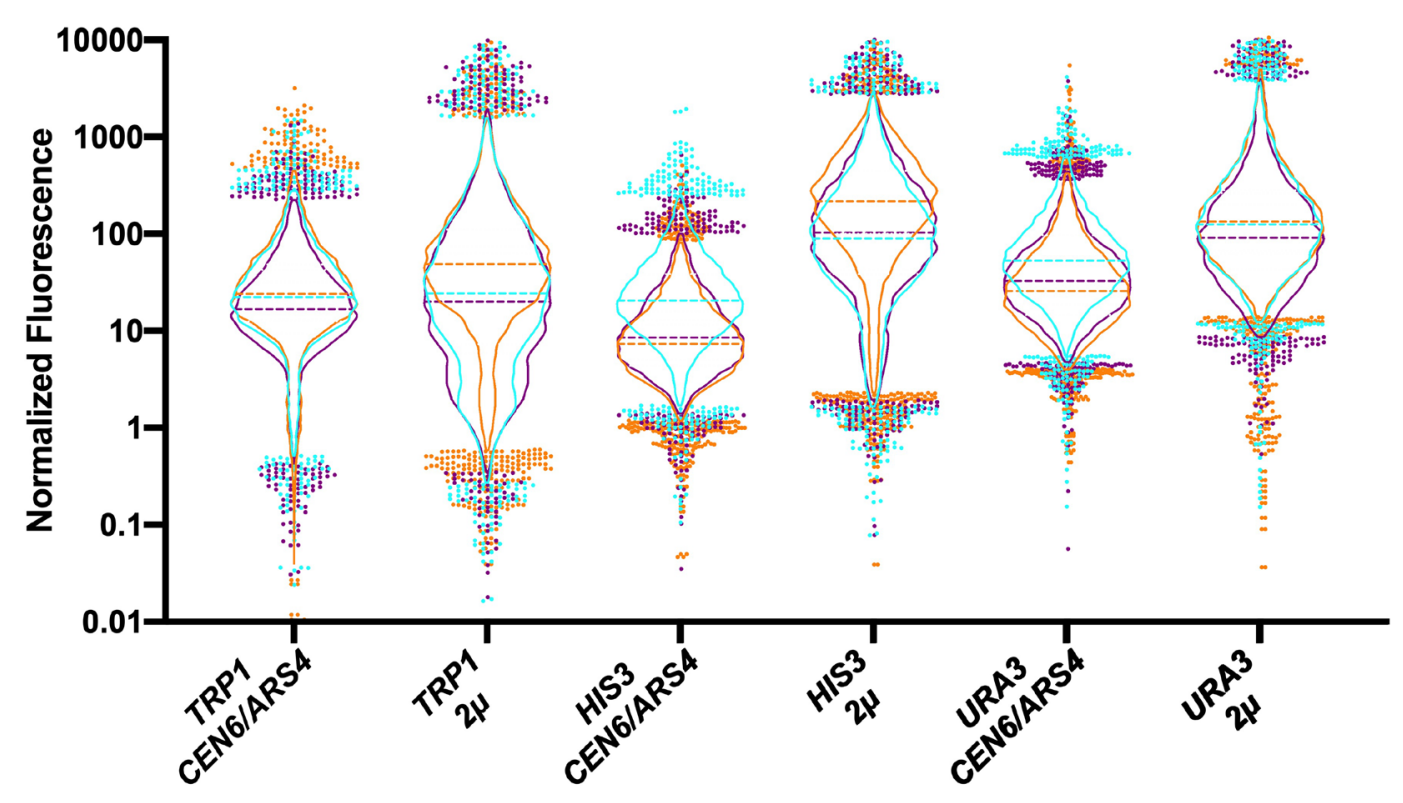

b

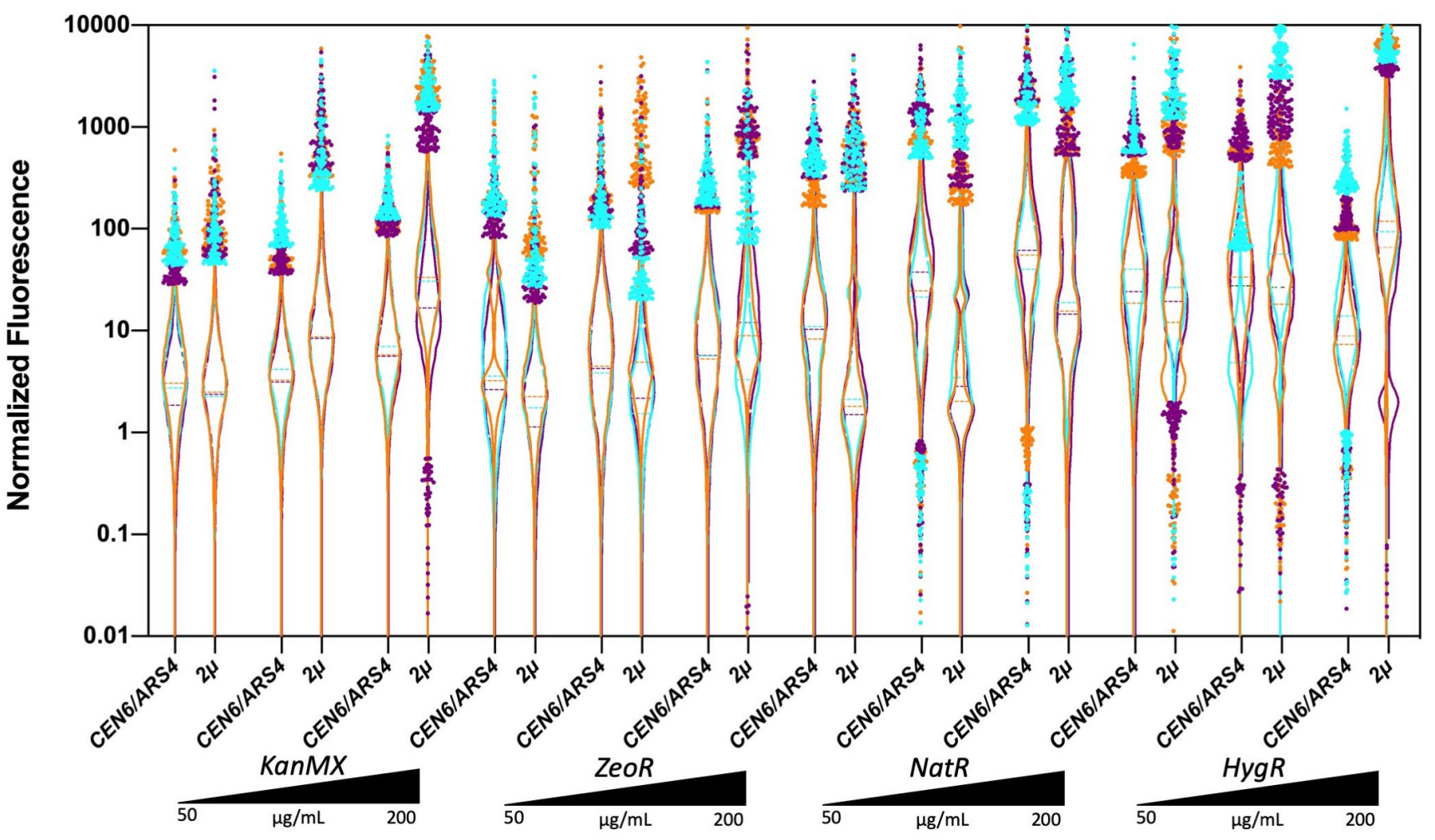

Figure S2 Characterization of selective markers and origins of replication. a) Violin plots of flow cytometry data for 3 auxotrophic markers and 2 origins of replication. b) Violin plots of flowcytometry data for 7 antifungal markers and 2 origins of replication tested in 3 different antifungal concentrations $(50,100,200 \mu \mathrm{g} / \mathrm{mL}$ ).

3 auxotrophic markers, 4 antifungal markers and 2 origins of replications were cloned behind TDH3 promoter, mRuby2 fluorescent protein and TDH1 terminator. Cyan, orange and purple represent first, second and third biological replicates, respectively. The dots represent the highest and lowest 2 percent of the cell population. Each flow cytometry run consists of 500010000 events. 


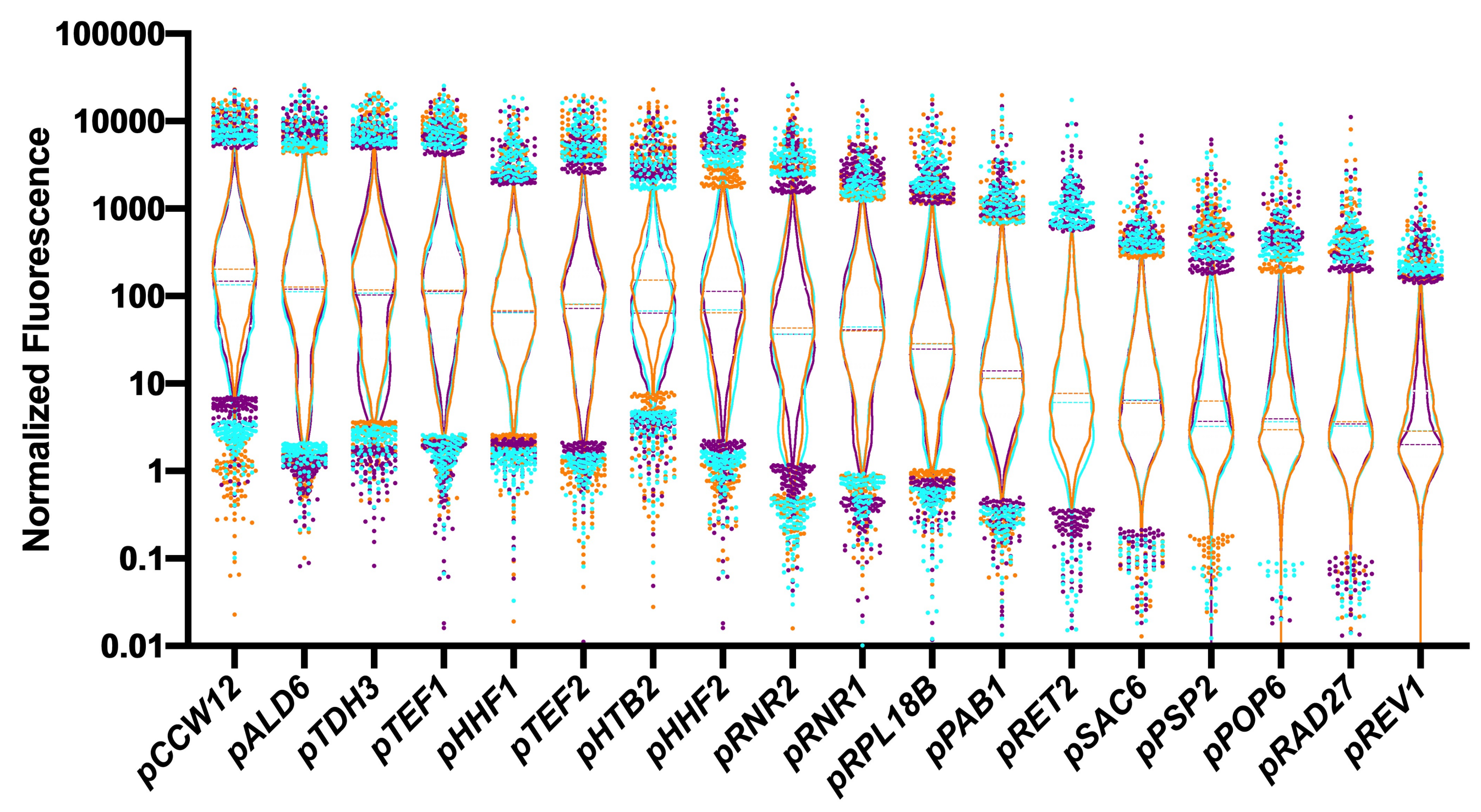

Figure S3 Characterization of Promoters. Violin plots of flowcytometry data for 18 constitutive promoters. 18 constitutive promoters were cloned in front of mRuby2 fluorescent protein and $t T D H 1$ terminator.

Cyan, orange and purple represent first, second and third biological replicates, respectively. The dots represent the $\% 2$ percent of the cell population. Each flowcytometry run consists of 10000 events. 


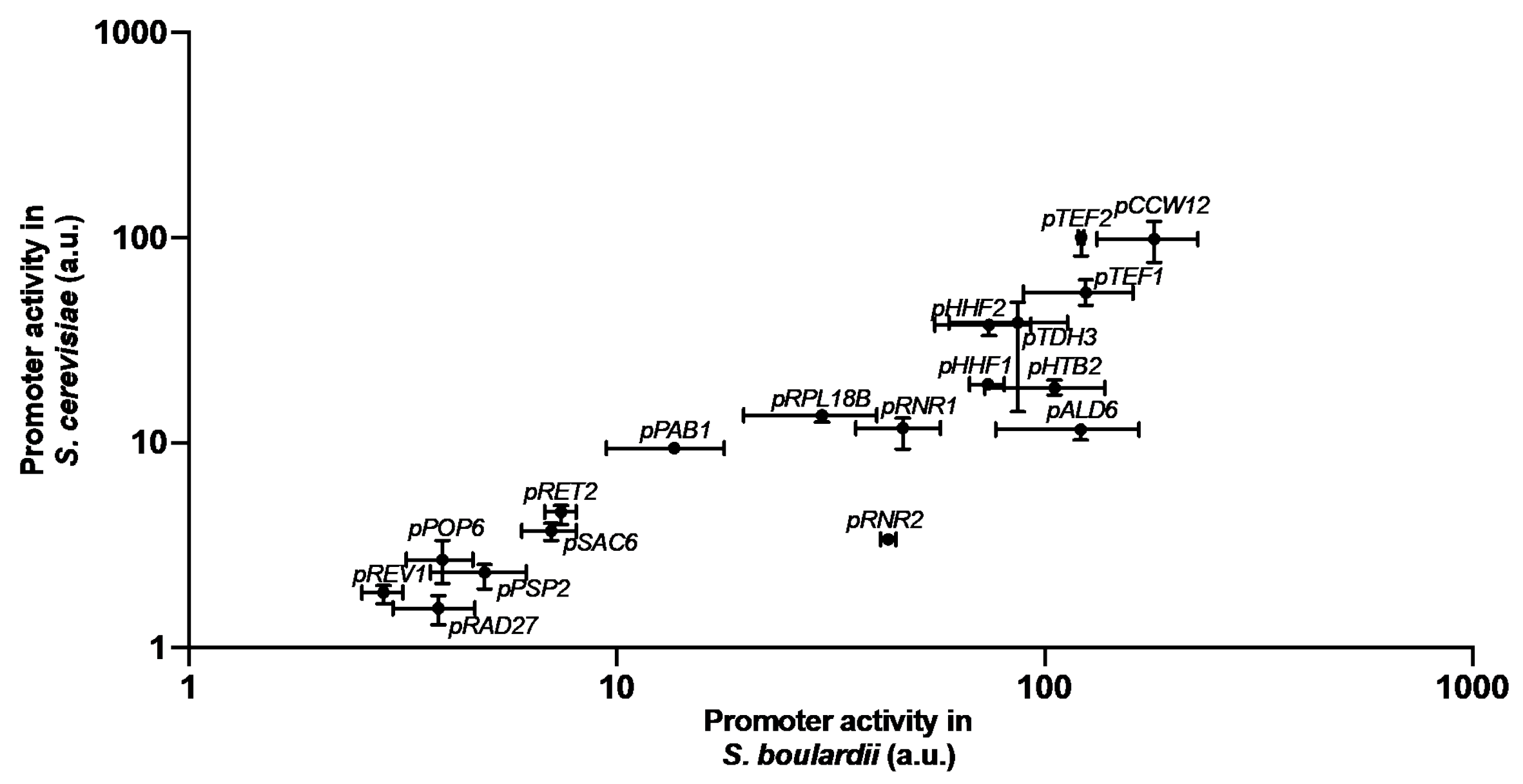

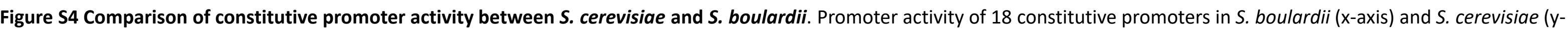

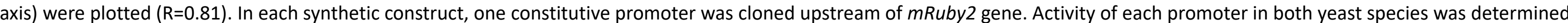

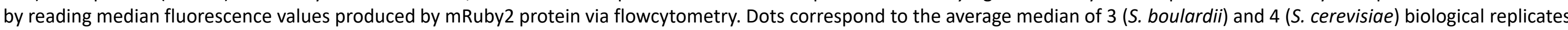
and the bars correspond to maximum and minimum median value obtained by the replicates. 


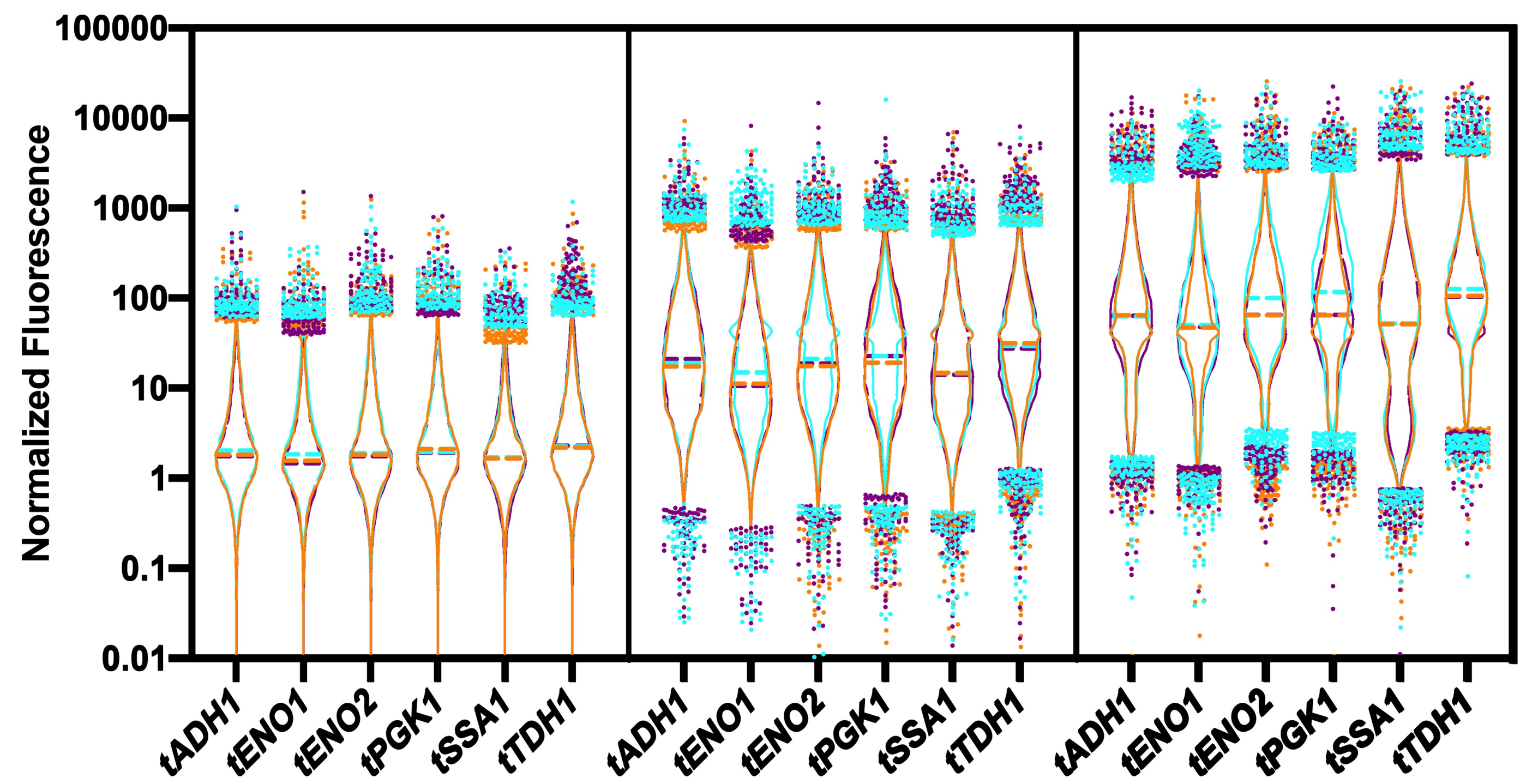

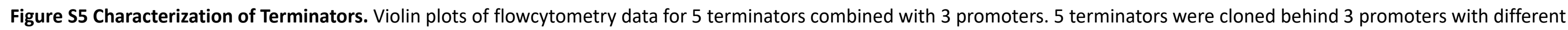
strength ( $p T D H 3$-strong-most right panel, $p R N R 1$-medium-middle panel, $p R E V 1$-weak-most left panel) and mRuby2 fluorescent protein.

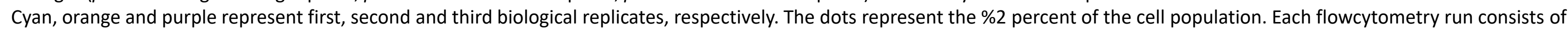
10000 events. 


\section{-}

$3 \mathrm{~kb}$

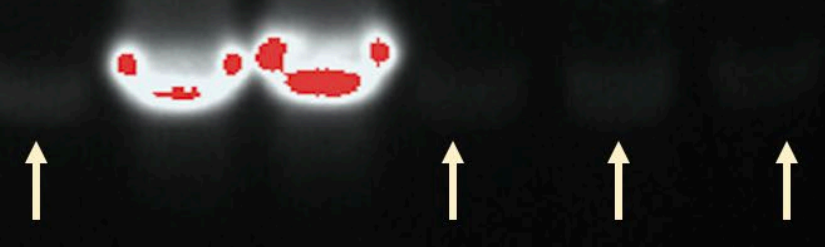

$1 \mathrm{~kb}$

Figure S6. Colony PCR of S. boulardii genome editing using only homologous recombination. The gel image shows that when only donor DNA is transformed into $S$. boulardii, the cells keeps a copy of the original chromosomal locus sequence. Lanes 1-6 lanes show a PCR for correct integration into INT1. Lanes 7-12 show a PCR for correct integration into INT4. In both cases, a correct integration will yield a PCR product of $3.8 \mathrm{~kb}$, while the native sequence yields a PCR product of $2.0 \mathrm{~kb}$. 


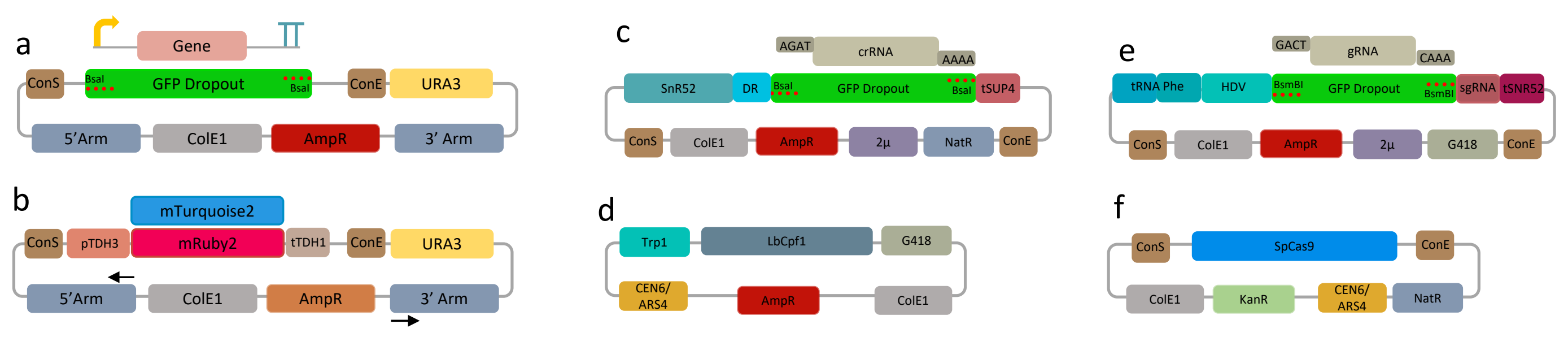

Figure S7. Genome editing plasmids. a. Vector for assembling the promoter, gene and terminator of interest. b. Example of constructed integration plasmid using cloning vector from a. c. Cloning vector with GFP dropout for constructing the CRISPR/LbCas12a crRNA d. CRISPR/LbCas12a expression vector. e. Cloning vector with GFP dropout for constructing the CRISPR/SpCas9 gRNA. f. CRISPR/SpCas9 expression vector. All GFP dropout vectors vectors are used in Golden Gate reactions with either Bsal or BsmBI enzymes. 


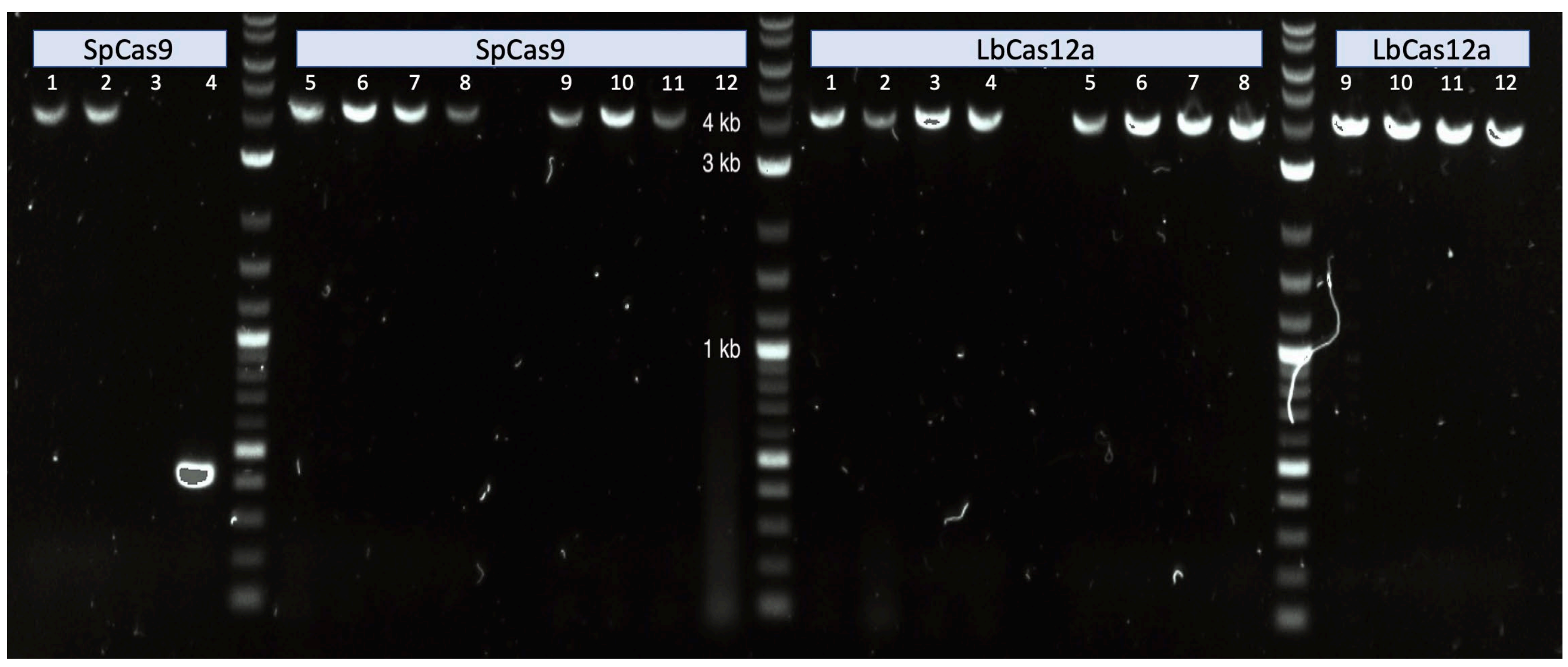

Figure S8. Colony PCR of $S$. boulardii genome editing using CRISPR/SpCas9 and CRISPR/LbCas12a. SpCas9 lanes 1-4 show a PCR screen for integration into INT1 (line 3 is failed PCR reaction and line 4 is unedited site), SpCas 9 lanes 5-8 show a PCR screen for integration into INT4, and SpCas9 lanes 9-12 show a PCR screen for integration into INT5. LbCas 12 a lanes 1-4 show a PCR screen for integration into INT1, LbCas12a lanes 5-8 show a PCR screen for integration into INT4 and LbCas12a lanes 9-12 show a PCR screen for integration into INT5. 


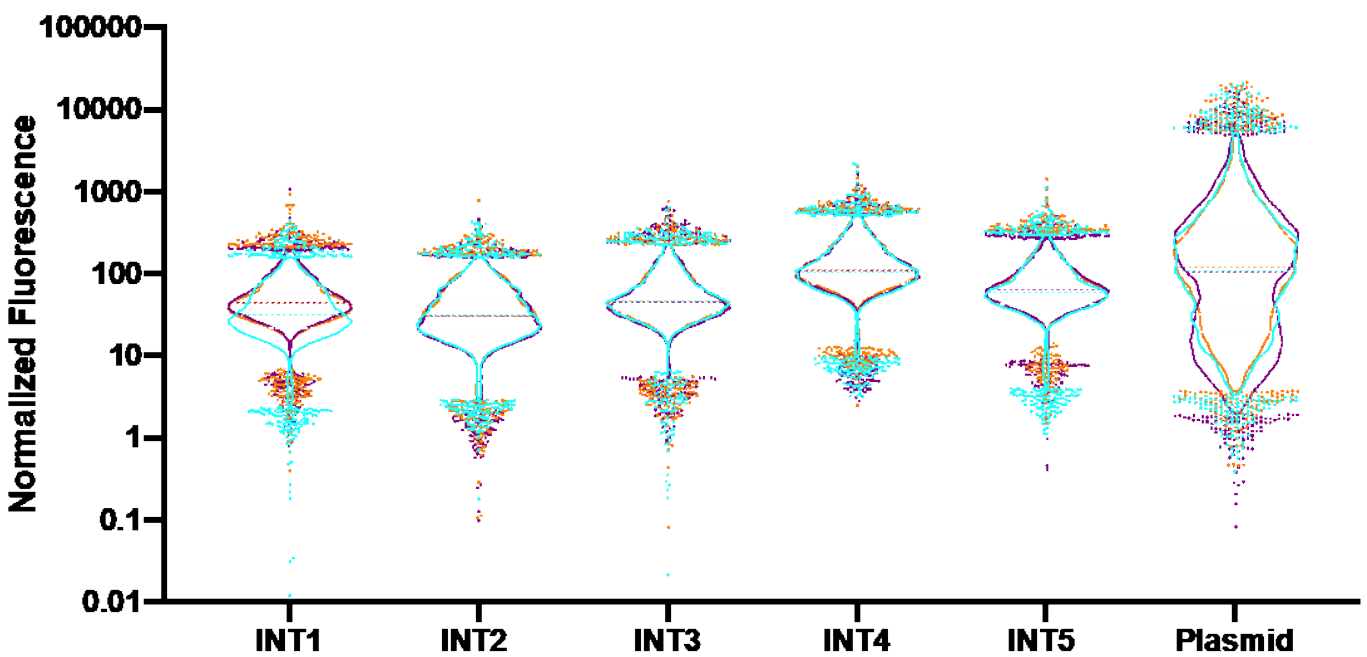

b

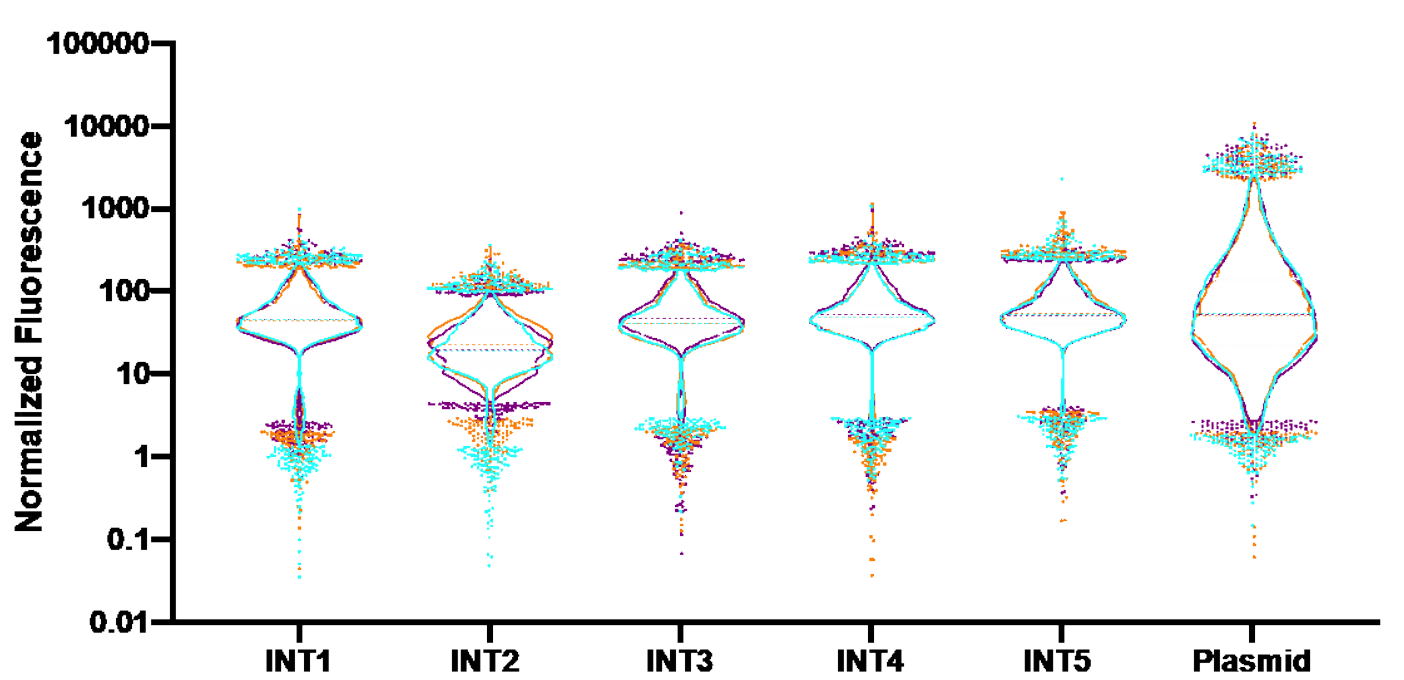

Figure S9 Effect of chromosomal locus on the expression of the fluorescent genes $\mathbf{m R u b y 2}$ and $\mathbf{m}$ Turqouise2. . Violin plots of flowcytometry data for a. $m$ Ruby 2 expression in 5 different loci in the genome compared to the expression of the same construct in plasmid with $2 \mu$. b. mTurqiouse 2 expression in 5 different loci in the genome compared to the expression of the same construct in plasmid with $2 \mu$ origin. constitutive promoters. 


$\begin{array}{rcccccc}1 & 2 & 3 & 4 & 5 & 6 \\ 7 & \bigcirc & & & \bigcirc & \bigcirc & \bigcirc \\ 7 & 8 & 9 & 10 & 11 & 12 \\ 0 & \bigcirc & 0 & \bigcirc & \bigcirc & \bigcirc \\ 13 & 14 & 15 & 16 & 17 & 18 \\ \bigcirc & \bigcirc & \bigcirc & \bigcirc & & \bigcirc \\ 19 & 20 & 21 & 22 & 23 & 24 \\ \bigcirc & \odot & & \bigcirc & \bigcirc & \bigcirc\end{array}$

Figure S10. in vivo Combinatorial Pathway Assembly in S. boulardii. a\&b) S. boulardii Transformation plates for violacein (a) and $\beta$-carotene (b) pathways. Violacein (c) and $\beta$-carotene (d) isolates picked from plates a \&b.

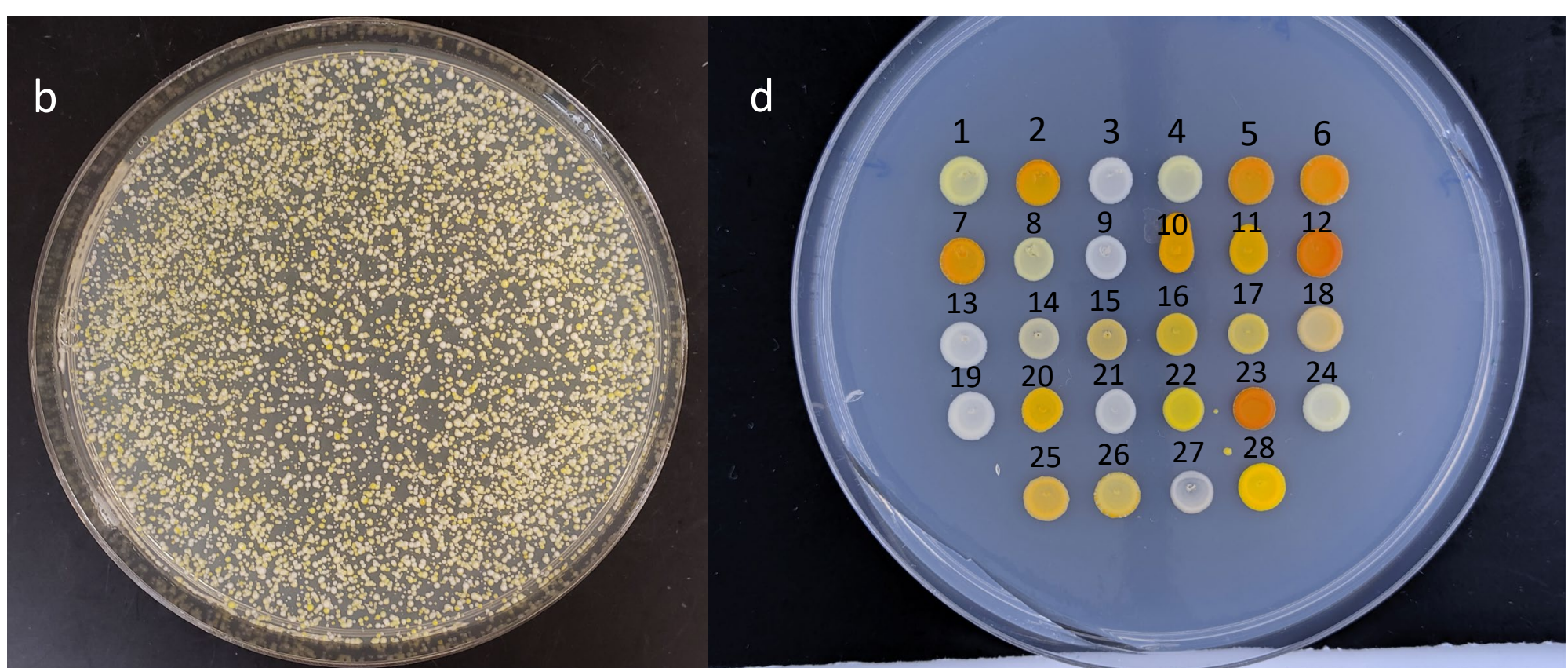




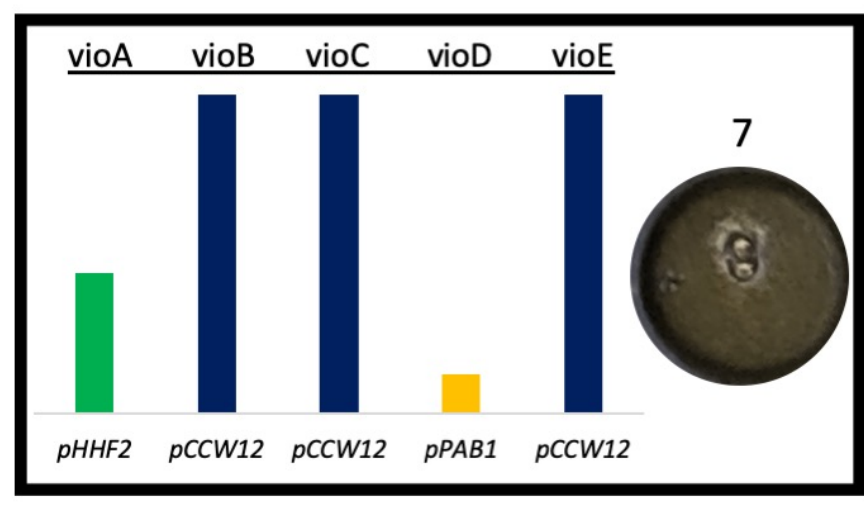

PPAB1 PHHF2 pHHF2 pCCW12pCCW12

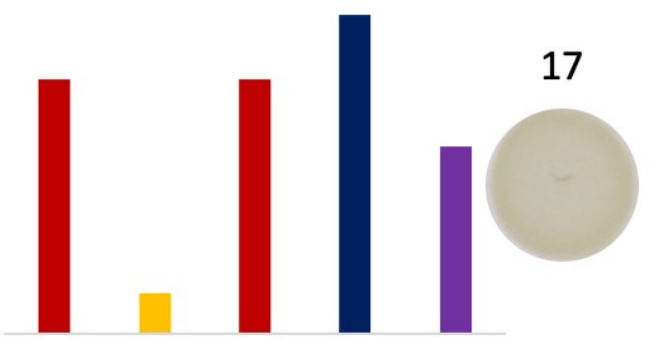

PTDH3 $\quad$ PHHF2 PCCW12 PHHF2 pHHF1

14

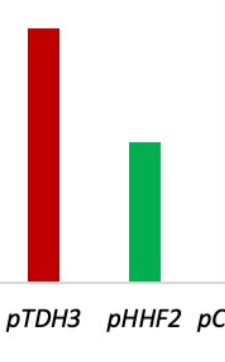

$$
\text { p }
$$

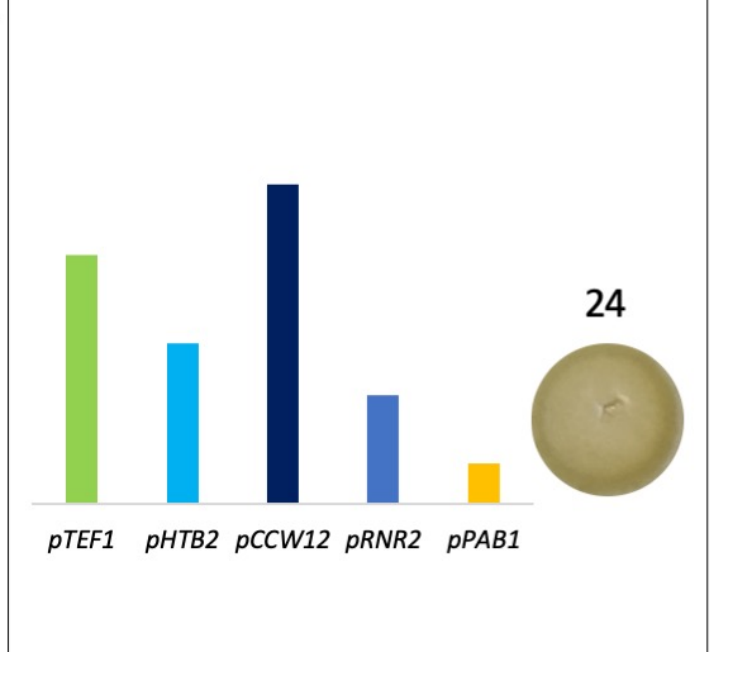

рTDH3 рPAB1

Figure S11. Combinatorial assembly of violacein pathway assembly in S. boulardii. Violacein pathway consisting of 5 genes (vioA, vioB, vioC, vioD and vioE) were assembled similar to $\beta$-carotene pathway. 8 out of 24 isolates (Supplementary Figure S8) were selected to proceed with sequencing based on stability of colony color after 3 serial passages. Sanger sequencing was done in a similar manner to the $\beta$-carotene isolates. Numbers on the colonies corresponds to the colony number from isolate plates on Figure S8c. The bar heights represent the normalized fluorescence values in linear scale for each promoter according to promoter characterization work (Figure 1a) 
b

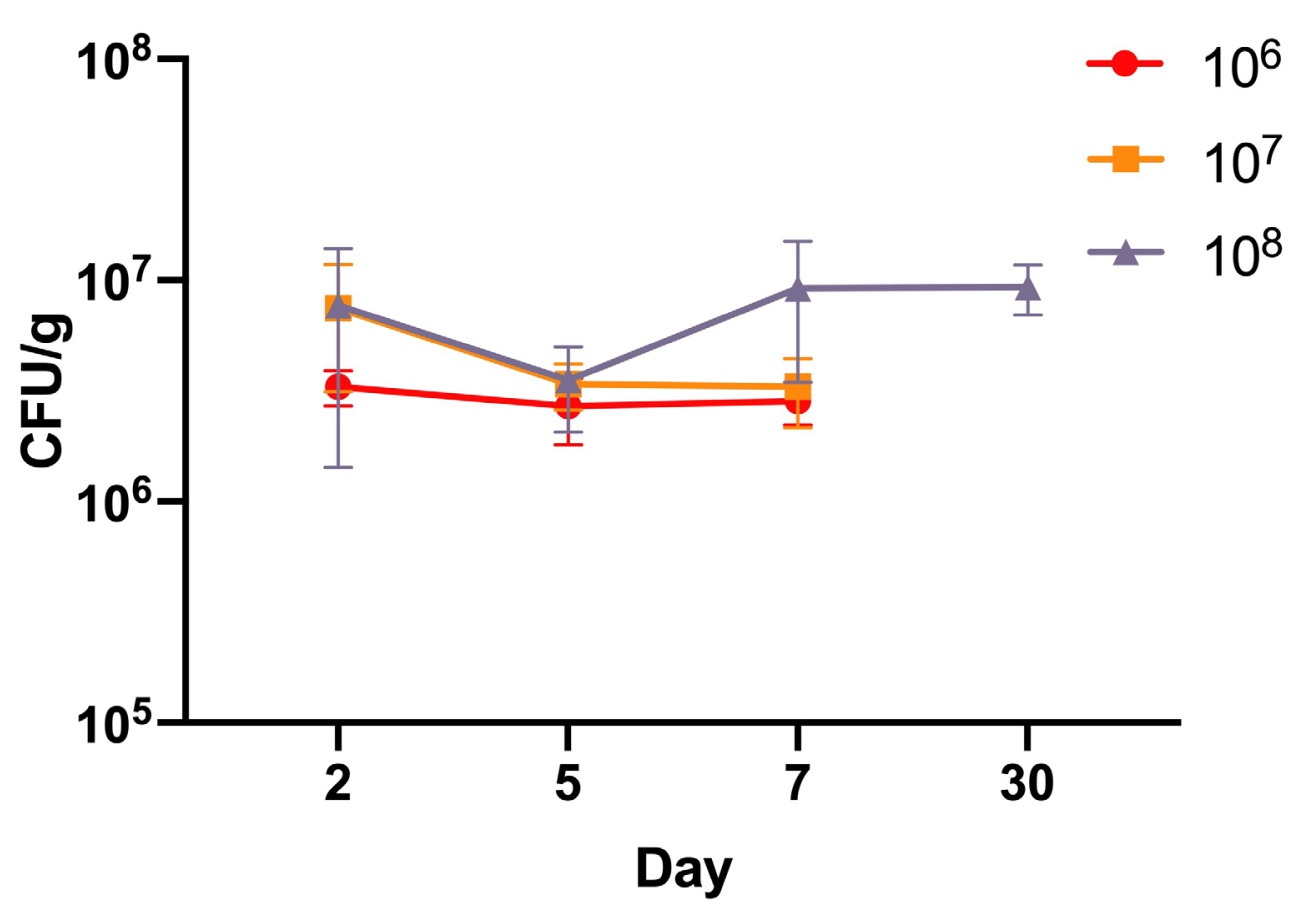

Figure S12. Dosage impacts the residence time of $S$. boulardii in germfree mice. a. germfree mice were gavaged with 3 different amounts of $S$. boulardii $\left(10^{6}, 10^{7}\right.$ and $10^{8}$ cells) on day 0 . b. Residence time of $S$. boulardii in germfree mice. Fecal samples were collected as shown in the timeline. Error bars indicate the standard deviation observed among 2 germ free mice for $10^{6} \mathrm{CFU}$ treatment, 8 germ free mice for $10^{7} \mathrm{CFU}$ treatment, and 4 germ free mice for $10^{8} \mathrm{CFU}$ treatment In all mouse models. Feces were collected prior to Sb gavage during days when both occurred. 


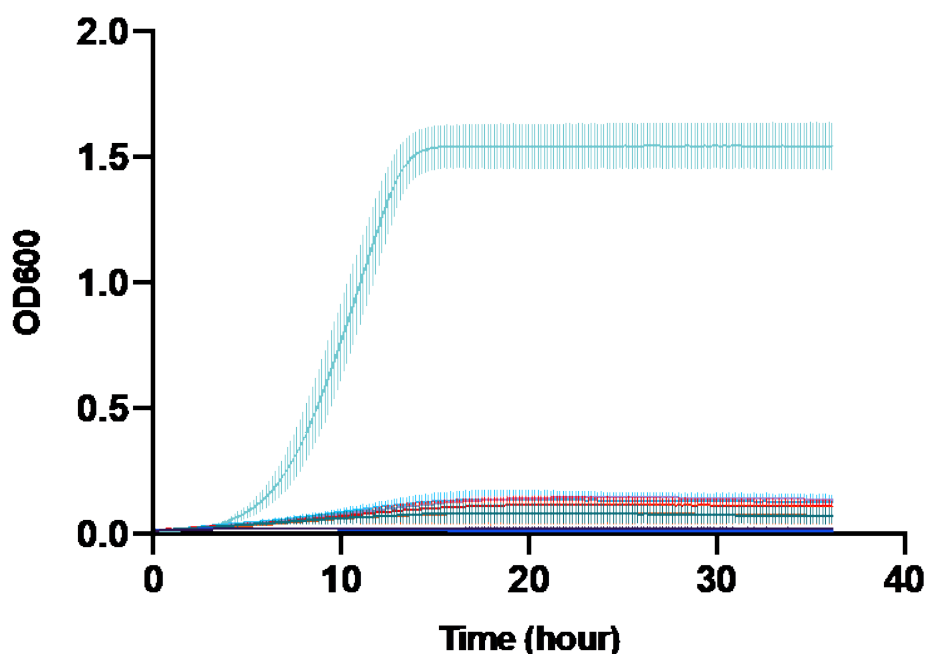

- $200 \mu \mathrm{g} / \mathrm{ml}$

- $100 \mu \mathrm{g} / \mathrm{ml}$

- $50 \mu \mathrm{g} / \mathrm{ml}$

- $25 \mu \mathrm{g} / \mathrm{ml}$

- $12.5 \mu \mathrm{g} / \mathrm{m}$

- $6.25 \mu \mathrm{g} / \mathrm{ml}$

- $3.125 \mu \mathrm{g} / \mathrm{ml}$

- $0 \mu \mathrm{g} / \mathrm{ml}$

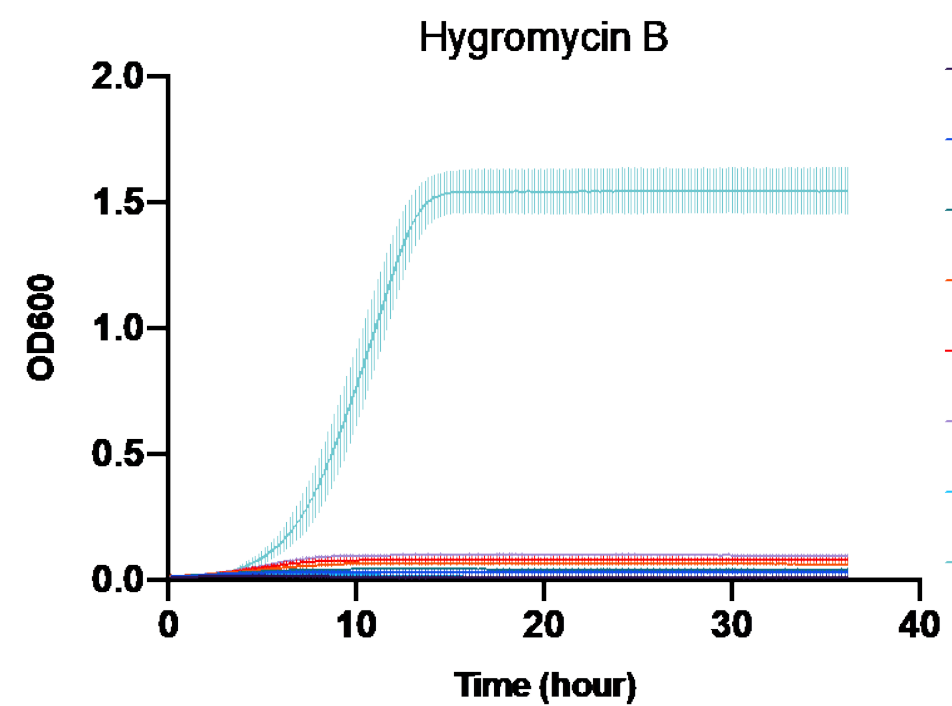

- $200 \mu \mathrm{g} / \mathrm{ml}$

- $100 \mu \mathrm{g} / \mathrm{ml}$

- $50 \mu \mathrm{g} / \mathrm{ml}$

- $25 \mu \mathrm{g} / \mathrm{ml}$

- $12.5 \mu \mathrm{g} / \mathrm{ml}$

- $3.125 \mu \mathrm{g} / \mathrm{m}$ $0 \mu \mathrm{g} / \mathrm{m}$
- $6.25 \mu \mathrm{g} / \mathrm{ml}$

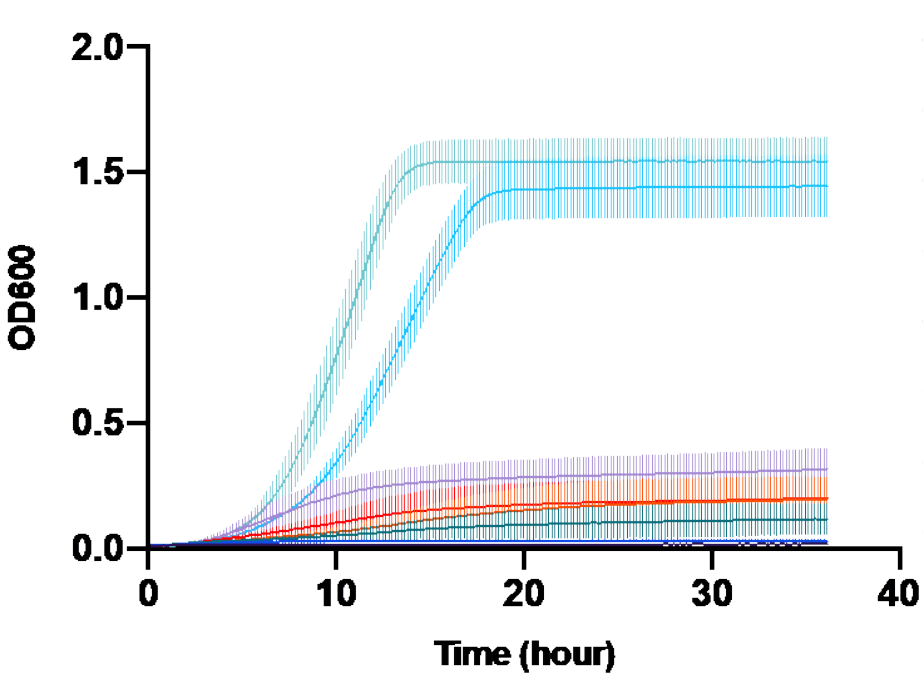

- $200 \mu \mathrm{g} / \mathrm{ml}$

- $100 \mu \mathrm{g} / \mathrm{ml}$

- $50 \mu \mathrm{g} / \mathrm{ml}$

- $25 \mu \mathrm{g} / \mathrm{ml}$

- $12.5 \mu \mathrm{g} / \mathrm{m}$

- $6.25 \mu \mathrm{g} / \mathrm{m}$

- $3.125 \mu \mathrm{g} / \mathrm{ml}$ $0 \mu \mathrm{g} / \mathrm{ml}$

Zeocin

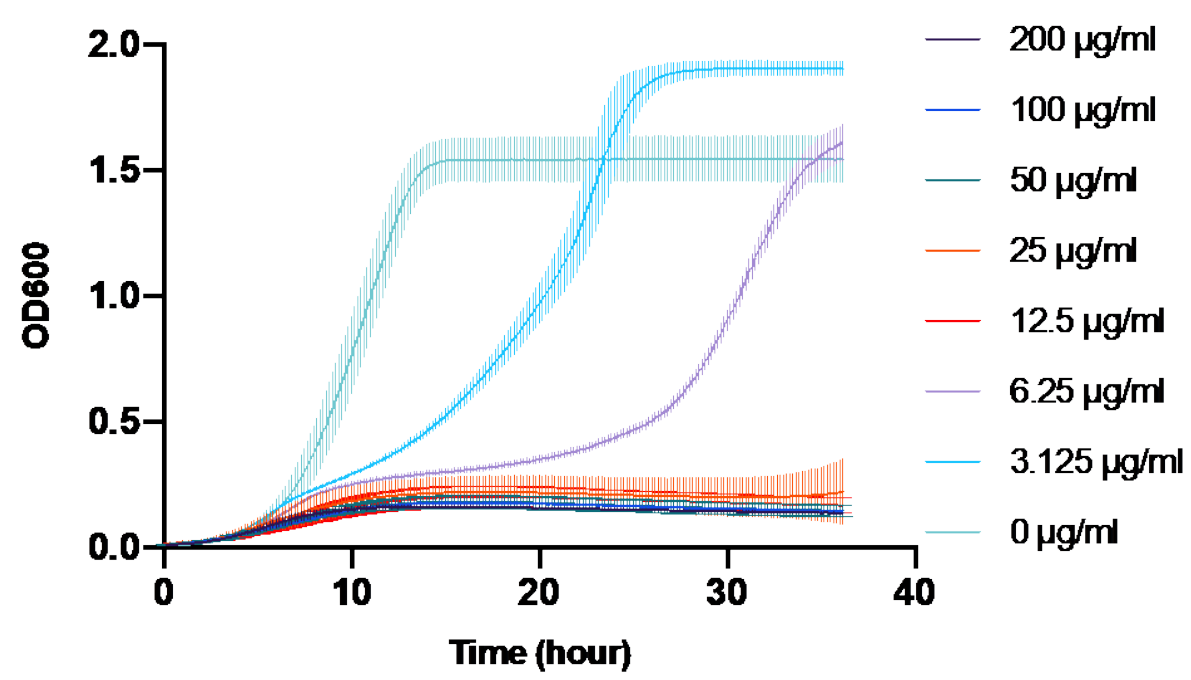

Figure S13. S. boulardii Minimum Inhibitory Concentration (MIC) Tests with Nourseothricin, G418 Geneticin, Hygromycin B and Zeocin. Error bars indicate the standard deviation observed among 3 biological replicates. 


\begin{tabular}{|c|c|c|c|c|c|}
\hline MoClo kit & Promoter & Score & Expect & Identities & Gaps \\
\hline YTK009 & $p T D H 3$ & 1218 bits(659) & 0 & $673 / 680(99 \%)$ & $0 / 680(0 \%)$ \\
\hline YTK010 & $p C C W 12$ & 1234 bits $(668)$ & 0 & $690 / 700(98 \%)$ & $4 / 700(0 \%)$ \\
\hline YTK011 & $p P G K 1$ & 1282 bits(694) & 0 & $698 / 700(99 \%)$ & $0 / 700(0 \%)$ \\
\hline YTK012 & $p H H F 2$ & 1197 bits(648) & 0 & $667 / 676(99 \%)$ & $1 / 676(0 \%)$ \\
\hline YTK013 & $p T E F 1$ & 1271 bits(688) & 0 & $696 / 700(99 \%)$ & $0 / 700(0 \%)$ \\
\hline YTK014 & $p T E F 2$ & 917 bits(496) & 0 & $534 / 550(97 \%)$ & $11 / 550(2 \%)$ \\
\hline YTK015 & $p H H F 1$ & 1254 bits(679) & 0 & $694 / 701(99 \%)$ & $1 / 701(0 \%)$ \\
\hline YTK016 & $p H T B 2$ & 1210 bits(655) & 0 & $683 / 696(98 \%)$ & $4 / 696(0 \%)$ \\
\hline YTK017 & $p R P L 18 B$ & 1236 bits(669) & 0 & $690 / 700(99 \%)$ & $2 / 700(0 \%)$ \\
\hline YTK018 & $p A L D 6$ & 1266 bits(685) & 0 & $695 / 700(99 \%)$ & $0 / 700(0 \%)$ \\
\hline YTK019 & $p P A B 1$ & 1271 bits(688) & 0 & $697 / 701(99 \%)$ & $1 / 701(0 \%)$ \\
\hline YTK020 & $p R E T 2$ & 1288 bits(697) & 0 & $699 / 700(99 \%)$ & $0 / 700(0 \%)$ \\
\hline YTK021 & $p R N R 1$ & 1266 bits(685) & 0 & $695 / 700(99 \%)$ & $0 / 700(0 \%)$ \\
\hline YTK022 & $p S A C 6$ & 1266 bits(685) & 0 & $695 / 700(99 \%)$ & $0 / 700(0 \%)$ \\
\hline YTK023 & $p R N R 2$ & 1251 bits(677) & 0 & $695 / 703(99 \%)$ & $4 / 703(0 \%)$ \\
\hline YTK024 & $p P O P 6$ & 1168 bits(632) & 0 & $688 / 712(97 \%)$ & $15 / 712(2 \%)$ \\
\hline YTK025 & $p R A D 27$ & 1184 bits(641) & 0 & $678 / 694(98 \%)$ & $9 / 694(1 \%)$ \\
\hline YTK026 & $p P S P 2$ & 1236 bits(669) & 0 & $689 / 699(99 \%)$ & $0 / 699(0 \%)$ \\
\hline YTK027 & $p R E V 1$ & 1251 bits(677) & 0 & $693 / 700(99 \%)$ & $4 / 700(0 \%)$ \\
\hline YTK028 & $p M F A 1$ & 1271 bits(688) & 0 & $697 / 701(99 \%)$ & $1 / 701(0 \%)$ \\
\hline
\end{tabular}




\begin{tabular}{|l|c|c|}
\hline Site & Chromosome & Locus \\
\hline INT 1 & $\mathbf{V}$ & YERC $\Delta 8$ \\
\hline INT 2 & VIII & YHRCA14 \\
\hline INT 3 & $\mathbf{X V}$ & non-coding region between NTR1(YOR071c) and GYP1 (YOR070c) \\
\hline INT 4 & $\mathbf{X I}$ & non-coding region between SRP40(YKR092C) and PTR2 (YKR093W) \\
\hline INT 5 & $\mathbf{X V I}$ & YPRCT3 \\
\hline
\end{tabular}

Table S2. Integration locations (INT1-INT5) in S. boulardii's genome. 


\begin{tabular}{|c|l|l|}
\hline INT site & \multicolumn{1}{|c|}{ SpCas9 guide } & \multicolumn{1}{|c|}{ LbCpf1 guide } \\
\hline \multirow{3}{*}{1} & ACTCCTGGGAGAGAACATTC & TCTTCCTTCCCGTTATACTTTGT \\
\cline { 2 - 3 } & AACTAGTATACCATCATATA & ATAATTGTTCGGATTGCATTGTT \\
\cline { 2 - 3 } & AATTCACAAAAGAGAATCGA & TGAATTCCTATAACGTCGGGAGA \\
\hline 2 & ACAAATGTCAGAAATATGGC & TCATACAACCAATGATTAATGTC \\
\hline 3 & TATTAGAACCAGGGAGGTCC & AAAGATCCATACTTCTCTACATT \\
\hline \multirow{3}{*}{4} & TTTCAACGCTTTCGAAGATG & TGTCAGATGTCATTATGGTCTCC \\
\cline { 2 - 3 } & GAATACTCAGACGTTTTCTG & CAGCCACATCTACAGGATGTTGA \\
\cline { 2 - 3 } & CAGAACCGTGACGCAATCTG & CCGGAAAAGCTAAGAGTTTTCTG \\
\hline \multirow{3}{*}{5} & GGCTAATATAATGGTGATTC & GCAACCCAAGACTCGGCATACCA \\
\cline { 2 - 3 } & GATCAATATAACAATGCGTC & ACGTTTCCTTTTGCAATAGGTAC \\
\cline { 2 - 3 } & ACAGTGCTGAAGCAGAGATA & GAATATATCTCTCCAATACAGCG \\
\hline
\end{tabular}

Table S3. Guide RNA sequences used for genome editing assisted with the CRISPR nucleases SpCas9 or LbCas12a 
a

\begin{tabular}{|c|c|c|c|}
\hline & \multicolumn{3}{|c|}{ Genome Editing Method } \\
\hline Site & SpCas9 & LbCas12a & DNA \\
\hline \multirow{3}{*}{ INT 1 } & $191 / 232$ & $399 / 424$ & $34 / 72$ \\
\cline { 2 - 4 } & $531 / 603$ & $186 / 199$ & $44 / 68$ \\
\cline { 2 - 4 } & $237 / 275$ & $397 / 401$ & $21 / 58$ \\
\hline \multirow{3}{*}{ INT 4 } & $526 / 627$ & $751 / 795$ & $54 / 98$ \\
\cline { 2 - 4 } & $588 / 679$ & $743 / 784$ & $62 / 81$ \\
\cline { 2 - 4 } & $299 / 358$ & $782 / 839$ & $46 / 73$ \\
\hline \multirow{3}{*}{ INT 5 } & $503 / 597$ & $679 / 716$ & $19 / 92$ \\
\cline { 2 - 4 } & $280 / 328$ & $632 / 662$ & $41 / 142$ \\
\cline { 2 - 4 } & $242 / 294$ & $729 / 764$ & $38 / 107$ \\
\hline
\end{tabular}

b

\begin{tabular}{|c|c|c|c|}
\hline & \multicolumn{3}{|c|}{ Genome Editing Method \% } \\
\hline Site & SpCas9 & LbCas12a & DNA \\
\hline \multirow{3}{*}{ INT 1 } & 82.3 & 94.1 & 47.2 \\
\cline { 2 - 4 } & 88.1 & 93.5 & 64.7 \\
\cline { 2 - 4 } & 86.2 & 99.0 & 36.2 \\
\hline Avg INT 1 & 85.5 & 95.5 & 49.4 \\
\hline \multirow{3}{*}{ INT 4 } & 83.9 & 94.5 & 55.1 \\
\cline { 2 - 4 } & 86.6 & 94.8 & 76.5 \\
\cline { 2 - 4 } Avg INT 4 & 83.5 & 93.2 & 63.0 \\
\hline \multirow{3}{*}{ INT 5 } & 84.7 & 94.1 & 64.9 \\
\cline { 2 - 4 } & 84.3 & 94.8 & 20.7 \\
\cline { 2 - 4 } & 85.4 & 95.5 & 28.9 \\
\hline Avg INT 5 & 82.3 & 95.4 & 35.5 \\
\hline
\end{tabular}

Table S4. S. boulardii genome editing efficiencies using 3 different methods. a. Number of colonies screened for each editing method. Successfully edited cells are defined by the number of fluorescent (mTurquoise2) colonies divided by the total number of colonies on the plate $\mathbf{b}$. The percentage of successfully edited cells in the 3 different locations using 3 different editing methods. 